\title{
Pulmonary Mycobacterium tuberculosis control associates with CXCR3- and CCR6- expressing antigen-specific Th1 and Th17 cell recruitment
}

\author{
Uma Shanmugasundaram, ${ }^{1}$ Allison N. Bucsan, ${ }^{2}$ Shashank R. Ganatra, ${ }^{2,3,4}$ Chris Ibegbu, ${ }^{1,5}$ \\ Melanie Quezada, ${ }^{1}$ Robert V. Blair, ${ }^{6}$ Xavier Alvarez, ${ }^{2,6}$ Vijayakumar Velu, ${ }^{1,5}$ Deepak Kaushal, ${ }^{2,3,4}$ \\ and Jyothi Rengarajan ${ }^{1,5,7}$ \\ ${ }^{1}$ Emory Vaccine Center, Emory University, Atlanta, Georgia, USA. ${ }^{2}$ Tulane National Primate Research Center, Tulane \\ University School of Medicine, Covington, Louisiana, USA. ${ }^{3}$ Southwest National Primate Research Center, San Antonio, \\ Texas, USA. ${ }^{4}$ Texas Biomedical Research Institute, San Antonio, Texas, USA. ${ }^{5}$ Yerkes National Primate Research Center, \\ Emory University, Atlanta, Georgia, USA. ${ }^{6}$ Division of Comparative Pathology, Tulane National Primate Research Center, \\ Covington, Louisiana, USA. 'Division of Infectious Diseases, Department of Medicine, Emory University School of \\ Medicine, Atlanta, Georgia, USA.
}

\begin{abstract}
Mycobacterium tuberculosis-specific (M. tuberculosis-specific) T cell responses associated with immune control during asymptomatic latent tuberculosis infection (LTBI) remain poorly understood. Using a nonhuman primate aerosol model, we studied the kinetics, phenotypes, and functions of $M$. tuberculosis antigen-specific T cells in peripheral and lung compartments of $M$. tuberculosis-infected asymptomatic rhesus macaques by longitudinally sampling blood and bronchoalveolar lavage, for up to 24 weeks postinfection. We found substantially higher frequencies of $M$. tuberculosis-specific effector and memory $\mathrm{CD4}^{+}$and $\mathrm{CD}^{+} \mathrm{T}$ cells producing IFN- $\gamma$ in the airways compared with peripheral blood, and these frequencies were maintained throughout the study period. Moreover, M. tuberculosis-specific IL-17+ and IL-17+IFN- $\gamma^{+}$double-positive T cells were present in the airways but were largely absent in the periphery, suggesting that balanced mucosal Th1/Th17 responses are associated with LTBI. The majority of $M$. tuberculosis-specific CD4 T cells that homed to the airways expressed the chemokine receptor CXCR3 and coexpressed CCR6. Notably, $\mathrm{CXCR3}^{+} \mathrm{CD}^{+}$cells were found in granulomatous and nongranulomatous regions of the lung and inversely correlated with $M$. tuberculosis burden. Our findings provide insights into antigenspecific $T$ cell responses associated with asymptomatic $M$. tuberculosis infection that are relevant for developing better strategies to control TB.
\end{abstract}

Conflict of interest: The authors have declared that no conflict of interest exists.

Copyright: ( 2020 , American Society for Clinical Investigation.

Submitted: March 10, 2020

Accepted: June 4, 2020

Published: July 23, 2020

Reference information: /CI Insight. 2020;5(14):e137858.

https://doi.org/10.1172/jici.

insight.137858.

\section{Introduction}

After close contact with a person with active tuberculosis (TB), only a minority of individuals develop primary TB disease. The majority of individuals successfully control Mycobacterium tuberculosis ( $M$. tuberculosis) infection in a clinically asymptomatic state termed latent TB infection (LTBI) (1). Individuals with LTBI are defined as having a positive tuberculin skin test (TST) and/or Interferon-gamma Release Assay (IGRA), a normal chest radiograph, and the absence of clinical signs and symptoms of disease (2). Latently infected individuals are generally thought to contain $M$. tuberculosis within granulomatous lesions in the lung without completely eradicating bacteria, although direct evidence for the persistence of M. tuberculosis in human LTBI is lacking. Moreover, it is increasingly recognized that clinically asymptomatic individuals likely reflect a spectrum of infection outcomes, ranging from individuals who may have eliminated infection, to those with low levels of replicating or nonreplicating persistent bacteria, to individuals who may harbor actively replicating bacteria without exhibiting overt clinical symptoms (3-5). Identifying immune responses associated with asymptomatic $M$. tuberculosis infection states will provide key insights into mechanisms of immune control that protect against progressing to active TB disease. 
Antigen-specific $\mathrm{T}$ cell responses are critical for immune control of $M$. tuberculosis infection. In response to $M$. tuberculosis infection, the majority of infected people mount robust $\mathrm{CD}^{+} \mathrm{T}$ cell responses involving Th1 cytokines, such as IFN- $\gamma$ and TNF- $\alpha$, which are important for activating macrophages and curtailing M. tuberculosis replication in the lung $(6,7)$. In addition, IL-17 and Th17 responses have emerged as important for protective immunity against TB $(8,9)$. Animal studies have shown a role for IL-17 in induction of chemokines, recruitment of $\mathrm{CD} 4^{+} \mathrm{T}$ cells to the site of infection, formation of granulomas, and protection during $M$. tuberculosis infection and Bacille Calmette-Guérin (BCG) vaccination (10-18). The role of IL-17 and Th17 responses in human TB is less clear and has been mainly studied by comparing individuals with active TB and healthy controls. Reports from humans vary widely, with studies showing no difference in the levels of IL-17 between the groups (19), while others have seen low levels of IL-17 in patients with TB compared with healthy controls $(20,21)$. Human genetic mutations and polymorphisms in IL-17 have been associated with TB susceptibility $(12,22)$, whereas other studies have shown the association of Th17/IL-17 responses with TB pathogenesis and disease progression (23-26). Overall, how IL-17, and in particular, M. tuberculosis antigen-specific Th17 cells, function to control $M$. tuberculosis infection during asymptomatic LTBI in humans remains poorly understood. We have limited knowledge of the onset and maintenance of $M$. tuberculosis antigen-specific Th1 and Th17 cell responses in the blood and lung compartments during LTBI and of the phenotypes and functions associated with the LTBI state. This is in part because small-animal models do not reproduce key aspects of human LTBI. Moreover, accurately documenting $M$. tuberculosis exposure, initial infection, and early events following infection in humans is almost impossible. Thus, studies of $M$. tuberculosis antigen-specific T cells in humans have been largely confined to cross-sectional characterization of peripheral responses in the blood (27-31). While some studies have examined responses in bronchoalveolar lavage (BAL) (32-34), longitudinal studies in humans comparing M. tuberculosis antigen-specific T cell responses in blood and lung compartments have been lacking. Thus, detailed characterization of the nature and kinetics of $M$. tuberculosis antigen-specific $T$ cells associated with human-like asymptomatic LTBI is important for identifying correlates of immune control and protection.

Nonhuman primate (NHP) macaque models of $M$. tuberculosis infection recapitulate multiple features of human $M$. tuberculosis infection, including clinically asymptomatic infection and symptomatic active TB disease (35-42), and are attractive for studying immune parameters associated with control of $M$. tuberculosis infection in peripheral blood and lung compartments. We have previously established a model of LTBI in Indian rhesus macaques, in which low-dose aerosol infection with M. tuberculosis CDC1551 leads to the development of asymptomatic $M$. tuberculosis infection. In this model, approximately $80 \%$ of infected animals remain disease free for up to 6 months postinfection (38) while only approximately $20 \%$ progress to active TB disease. In this study, we characterized the nature, magnitude, and kinetics of $M$. tuberculosis antigen-specific $\mathrm{CD}^{+}$and $\mathrm{CD}^{+} \mathrm{T}$ cell responses during asymptomatic LTBI in rhesus macaques over approximately 24 weeks postinfection, by serially sampling blood and lung compartments in conjunction with intensive clinical monitoring. We found substantially higher frequencies of $M$. tuberculosis-specific effector and memory CD4 ${ }^{+}$ and $\mathrm{CD}^{+} \mathrm{T}$ cells producing IFN- $\gamma$ in the airways compared with peripheral blood; these were maintained throughout the 24-week study period. Moreover, M. tuberculosis-specific IL-17 ${ }^{+}$and IL-17/IFN- $\gamma$ double-positive T cells were present in the airways but were largely absent in peripheral blood. The majority of $M$. tuberculosis-specific $\mathrm{CD}^{+} \mathrm{T}$ cells that homed to the airways expressed the chemokine receptor CXCR3 and coexpressed CCR6. Notably, CXCR3 ${ }^{+} \mathrm{CD} 4^{+}$cells were also found in the lungs of animals with LTBI and active TB and were associated with lower $M$. tuberculosis burdens. Our findings provide new insights into antigen-specific $\mathrm{T}$ cell responses associated with the establishment and maintenance of asymptomatic infection.

\section{Results}

Experimental design and clinical characteristics of rhesus macaques with asymptomatic $M$. tuberculosis infection. Six animals with no clinical signs or symptoms of disease were studied over approximately 24 weeks following lowdose aerosol infection with $M$. tuberculosis CDC1551 (Figure 1A). These animals were IGRA ${ }^{+}$; had a median chest radiograph (CXR) score of 0.4 , denoting no pulmonary lesions; and maintained normal C-reactive protein (CRP) levels (Figure 1B), body weight (Supplemental Figure 1A; supplemental material available online with this article; https://doi.org/10.1172/jci.insight.137858DS1), and temperature (Supplemental Figure 1B). All animals except 1 had detectable bacteria upon plating BAL (Figure 1C), and 3 of these animals had detectable, albeit low (<4 logs), lung bacterial loads at necropsy (Figure 1D). Examination of H\&E-stained lung tissue at necropsy (at $\sim 24$ weeks postinfection) showed that animals harbored varying degrees of inflammatory 
A

Mtb CDC1551

Weeks

TST/PRIMAGRAM

Euthanasia

Chest X-ray

Blood and BAL

Lung tissue

B

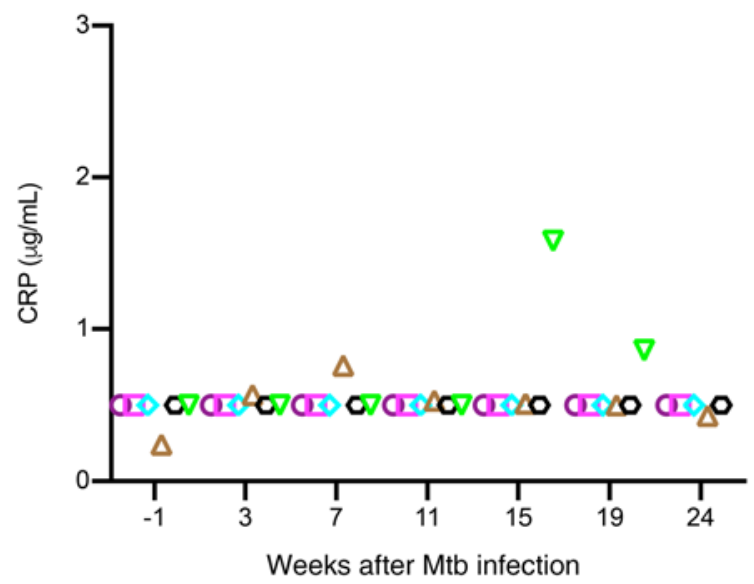

D

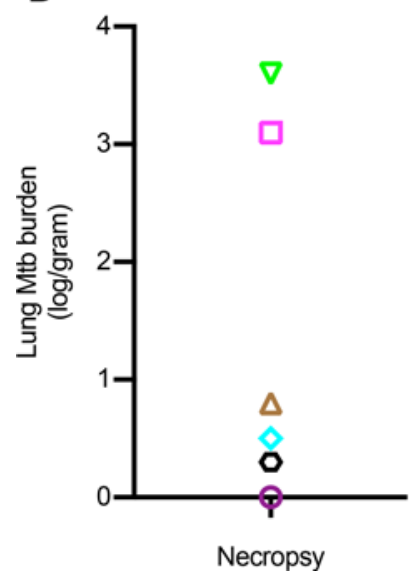

E

C

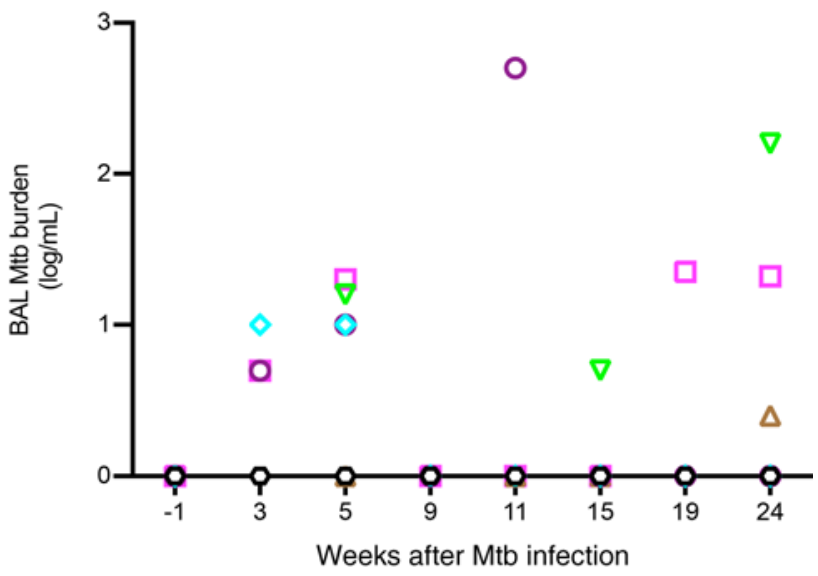

O JF47

口 HV02

$\diamond \quad$ JD72

$\triangle \quad$ JN75

- GP50

$\nabla \quad$ JK54

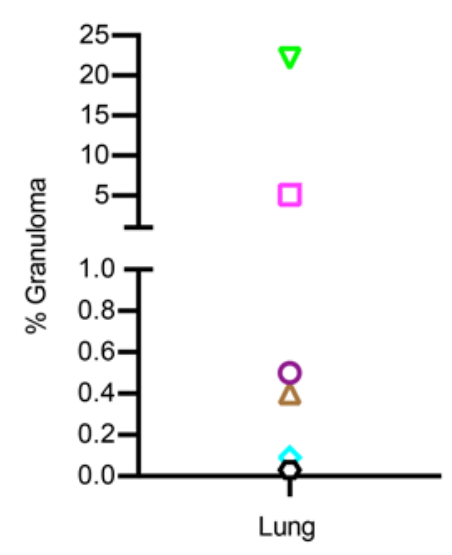

Figure 1. Experimental design and clinical characteristics of asymptomatic rhesus macaques with LTBI. (A) Eight rhesus macaques were infected by low-dose aerosol route with M. tuberculosis CDC1551 at week 0, and infection was confirmed at 3 and 7 weeks by TST and ICRA tests. Six macaques were defined as LTBI based on absence of clinical signs and symptoms of disease and a negative chest x-ray up to week 15 . Six animals remained asymptomatic and were longitudinally followed up until week 24. PBMCs and BAL were collected at indicated time points, and lung tissues were collected at necropsy. Each colored symbol represents an animal. (B) CRP levels were measured before infection (week -1) and at indicated time points postinfection for $n=$ 6 animals. (C) M. tuberculosis burden (CFU) in BAL measured at indicated weeks. (D) M. tuberculosis burden (CFU) in lung at necropsy (CFU per gram of tissue plated). (E) Percentage of granuloma in the lung tissue, determined by dividing the granulomatous area $\left(\mathrm{mm}^{2}\right)$ by the area of the annotated regions $\left(\mathrm{mm}^{2}\right)$ classified using an algorithm trained via a deep convolutional network (HALO AI).

lesions, ranging from $0.2 \%$ to $20 \%$ of the lung being constituted by granulomas (Figure 1E). These results highlight the heterogeneity of clinically asymptomatic animals that control M. tuberculosis infection, consistent with the idea that asymptomatic LTBI is represented by a spectrum of $M$. tuberculosis infection states $(3,5)$.

Comparison of total $C D 4^{+}$and $C D 8^{+} T$ cell frequencies in PBMCs and BAL of asymptomatic rhesus macaques. We assessed the frequencies of total $\mathrm{CD}^{+}$and $\mathrm{CD} 8^{+} \mathrm{T}$ cells before $M$. tuberculosis infection (week -1 ) and at $3,7,11,15,19$, and 24 weeks postinfection in the 6 animals with asymptomatic $M$. tuberculosis infection by intracellular cytokine staining (ICS) and flow cytometry (Figure 2 and Supplemental Figure 2). 
A

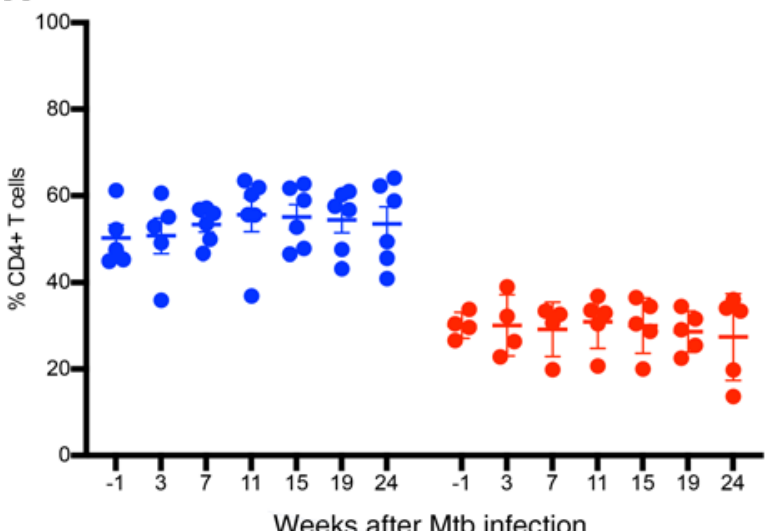

B

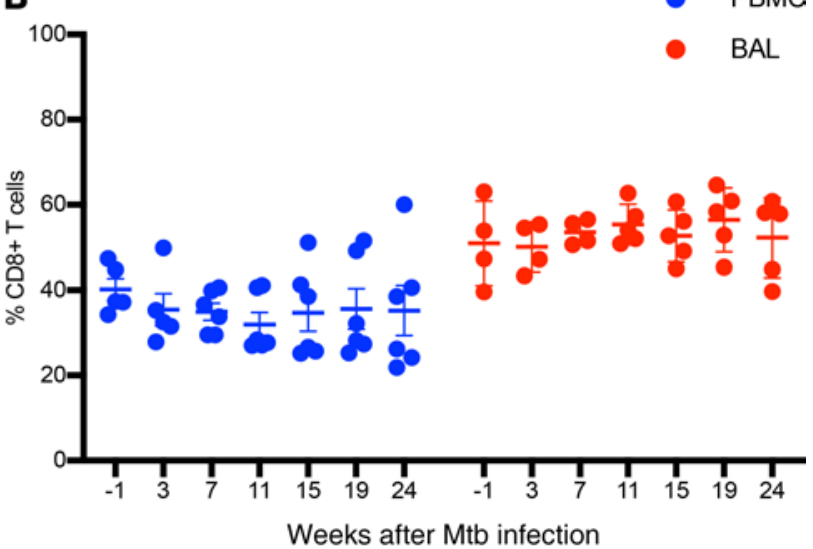

Figure 2. Frequencies of CD4+ and CD8+ $\mathbf{T}$ cells in BAL and PBMCs. Red and blue circles indicate BAL and PBMCs, respectively. (A) Higher frequencies of $\mathrm{CD}^{+} \mathrm{T}$ cells in PBMCs compared with BAL at all indicated time points $(P=0.01)$. (B) Higher frequencies of CD8 ${ }^{+}$T cells in BAL compared with PBMCs at all indicated time points $(P=0.01)$. Each data point represents an animal $(n=6)$, and horizontal lines indicate the mean with SEM. Wilcoxon's matched-pairs signed rank test was used to compare the frequencies of $\mathrm{CD} 4^{+}$and $\mathrm{CD} 8^{+} \mathrm{T}$ cells between BAL and PBMCs.

We observed significantly higher frequencies of $\mathrm{CD}^{+} \mathrm{T}$ cells in peripheral blood compared with BAL at all time points studied $(P=0.01)$. In contrast, higher frequencies of $\mathrm{CD} 8^{+} \mathrm{T}$ cells were present in BAL compared with peripheral blood at all time points $(P=0.01)$. Overall, $\mathrm{CD} 4^{+}$and $\mathrm{CD} 8^{+} \mathrm{T}$ cells were each maintained over time in both BAL and PBMCs during the study.

High frequencies of $M$. tuberculosis antigen-specific $C D 4^{+}$and $C D 8^{+} T$ cells producing IFN- $\gamma$ are maintained in the $B A L$ of rhesus macaques during asymptomatic $M$. tuberculosis infection. To assess the kinetics of $M$. tuberculosis antigen-specific $\mathrm{CD}^{+}$and $\mathrm{CD}^{+} \mathrm{T}$ cell responses in the peripheral blood and airways of asymptomatic animals that control $M$. tuberculosis infection as LTBI, we stimulated PBMC and BAL samples at each time point with $M$. tuberculosis cell wall antigens (CW) and ESAT-6/CFP-10 peptide pools, followed by ICS and flow cytometry to assess production of IFN- $\gamma$. In both PBMCs and BAL, CW- and ESAT-6/CFP-10-specific, IFN- $\gamma$-producing $\mathrm{CD}^{+}$(Figure $3, \mathrm{~A}$ and $\mathrm{B}$ ) and $\mathrm{CD}^{+}$(Figure $3, \mathrm{C}$ and $\mathrm{D}$ ) $\mathrm{T}$ cells were detected as early as 3 weeks post $-M$. tuberculosis infection. M. tuberculosis-specific $\mathrm{CD} 4^{+}$and $\mathrm{CD} 8^{+} \mathrm{T}$ cell frequencies increased at week 7 in both PBMCs and BAL and were maintained up to the necropsy endpoint. Overall, while frequencies of $M$. tuberculosis-specific IFN- $\gamma^{+} \mathrm{CD}^{+}{ }^{+}$were higher than their $\mathrm{CD}^{+}$counterparts, both $\mathrm{CD}^{+}$and $\mathrm{CD}^{+} \mathrm{T}$ cell frequencies were significantly $(P=0.03)$ higher in BAL compared with peripheral blood at all time points (mean \pm SEM, at week 7 after $M$. tuberculosis infection, $0.3 \% \pm 0.06 \%$ in PBMCs and $15 \% \pm 6.5 \%$ in $\mathrm{BAL}$ ). Thus, robust $M$. tuberculosis antigen-specific $\mathrm{CD} 4^{+}$and $\mathrm{CD} 8^{+} \mathrm{T}$ cell responses are maintained in lung compartments during asymptomatic LTBI.

High proportions of $C D 28^{+} C D 95^{+} M$. tuberculosis-specific memory T cells in peripheral blood and BAL. The proliferation of antigen-specific T cells in response to infection leads to development of a pool of antigen-experienced memory $\mathrm{T}$ cells that are important for mediating effective protection against rechallenge (43). TB vaccine strategies aim to elicit protective antigen-specific memory T cell responses $(44,45)$. Moreover, memory $\mathrm{T}$ cells are thought to play an important role in controlling $M$. tuberculosis infection during LTBI but remain poorly studied in lung compartments. We therefore investigated $M$. tuberculosis-specific effector and memory $\mathrm{CD}^{+}$and $\mathrm{CD}^{+} \mathrm{T}$ cell subsets in asymptomatic rhesus macaques by assessing their differentiation state, based on cell surface expression of CD28 and CD95 on IFN- $\gamma^{+} \mathrm{CD} 4^{+} \mathrm{T}$ cells and $\mathrm{CD}^{+} \mathrm{T}$ cells (46) (Figure 4 and Supplemental Figure 2). At week 7 postinfection we observed that antigenspecific IFN $-\gamma^{+} \mathrm{CD}^{+}$and $\mathrm{CD} 8^{+} \mathrm{T}$ cells in both PBMCs and BAL were predominantly CD28 ${ }^{+} \mathrm{CD} 5^{+}$(Figure $4, \mathrm{~A}-\mathrm{D})$, indicating a central memory-like phenotype. Moreover, these memory $\mathrm{T}$ cells were maintained at high levels throughout the time course of the study. While we observed higher proportions of antigen-specific CD28-CD95 ${ }^{+}$effector $\mathrm{CD} 8^{+} \mathrm{T}$ cells compared with $\mathrm{CD} 4^{+} \mathrm{T}$ cells in the BAL (Figure 4, C and $\mathrm{D}$ ), the relative proportions of $\mathrm{CD} 28^{+} \mathrm{CD} 95^{+}$and $\mathrm{CD} 28-\mathrm{CD} 95^{+} \mathrm{CD} 4^{+}$and $\mathrm{CD} 8^{+} \mathrm{T}$ cell subsets were maintained throughout latent infection in both peripheral blood and airways (Figure 4, E-H). Our data suggest that antigen-specific memory $\mathrm{T}$ cells in the blood and lung compartments are long-lived and are likely to contribute toward maintaining immune control during LTBI. 

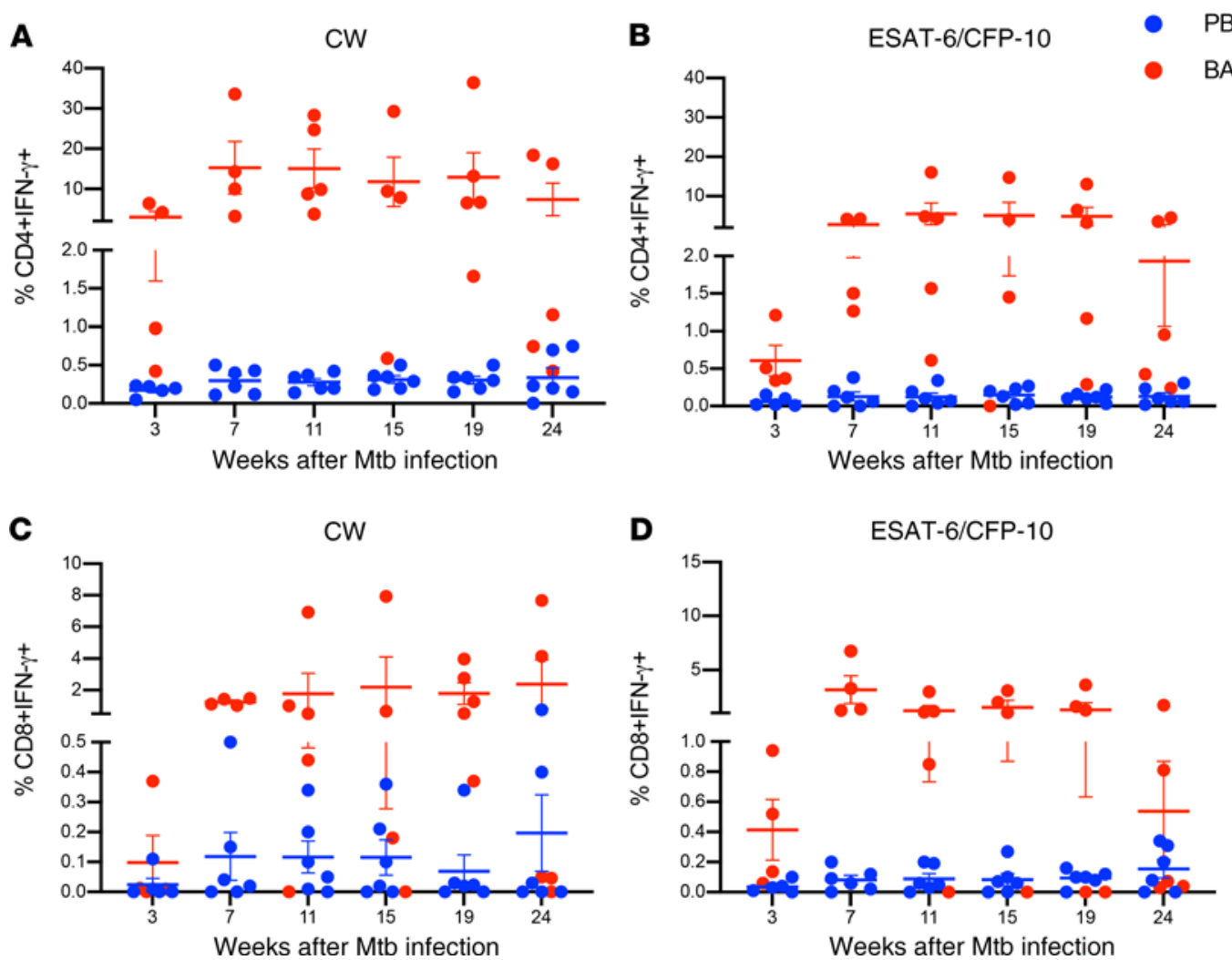

Figure 3. Kinetics of $M$. tuberculosis-specific CD4 ${ }^{+}$and $\mathbf{C D 8}{ }^{+}$T cells producing IFN- $\gamma$ in PBMCs and BAL. PBMCs (blue circles) and BAL (red circles) were stimulated with CW and ESAT-6/CFP-10 peptide pools, and IFN- $\gamma$ production by CD4 ${ }^{+}$and $C D 8^{+} T$ cells was assessed by ICS and flow cytometry. IFN- $\gamma-$ producing CD4 ${ }^{+}$ (A and $\mathbf{B})$ and $\mathrm{CD}^{+}(\mathbf{C}$ and $\mathbf{D})$ T cells at indicated time points (weeks) post-M. tuberculosis infection in macaques $(n=6)$ with $\mathrm{LTBI}^{\text {. CW-specific CD4 }}{ }^{+}(\mathbf{A})$ and $\mathrm{CD} 8^{+}$ (C) T cell frequencies and ESAT-6/CFP-10-specific CD4+ (B) and CD8 ${ }^{+}$(D) T cell frequencies in PBMCs and BAL are shown. Horizontal lines indicate the mean with SEM. Wilcoxon's matched-pairs signed rank test was used to compare the frequencies of IFN- $\gamma$-producing CD4 $4^{+}$and CD8 ${ }^{+}$T cells between BAL and PBMCs.

$I L-17^{+}$and IFN- $\gamma^{+} I L-17^{+}$T cells are present in the BAL but not in PBMCs from asymptomatic rhesus macaques. In addition to IFN- $\gamma$ production, IL-17 and Th17 responses have emerged as important for protective immunity against TB. To determine whether $M$. tuberculosis-specific IL-17-producing T cells are present during LTBI, we next assessed the kinetics of $\mathrm{IL}-17^{+} \mathrm{CD} 4^{+}$and $\mathrm{CD} 8^{+} \mathrm{T}$ cells in both BAL and PBMCs (Figure 5, A-D). We observed minimal to no $M$. tuberculosis-specific $\mathrm{IL}-17^{+} \mathrm{CD} 4^{+}$and $\mathrm{CD} 8^{+} \mathrm{T}$ cell responses in PBMCs throughout the time course of the study (Figure 5, A-D). In contrast, CW- and ESAT-6/CFP10-specific IL-17 ${ }^{+} \mathrm{CD}^{+}$ (Figure 5, A and B) and $\mathrm{CD}^{+}$cells (Figure 5, C and D) T cells were clearly detectable in the BAL. Thus, the presence of both $\mathrm{CD}^{+}$and $\mathrm{CD}^{+} M$. tuberculosis-specific $\mathrm{T}$ cells producing IL-17 in the airways demonstrates that mucosal IL-17-producing T cells accumulate at sites of $M$. tuberculosis infection and replication.

Although IL-17 is a hallmark of Th17 cells, IL-17/IFN- $\gamma$ double-positive T cells are also known to be present at mucosal sites of inflammation (16). We sought to investigate whether $M$. tuberculosis-specific $\mathrm{CD}^{+} \mathrm{T}$ cells that express both IFN- $\gamma$ and IL-17 are present in BAL (Figure 6A). At week 7 post $-M$. tuberculosis infection, in addition to CW- and ESAT-6/CFP-10-stimulated CD4 ${ }^{+} \mathrm{T}$ cells that singly expressed IFN- $\gamma$ or IL-17 (Figure 6, A-C), we found M. tuberculosis-specific T cells that coexpressed IFN- $\gamma$ and IL-17 (Figure 6D and Supplemental Figure 3, A-D). While these IFN- $\gamma /$ IL-17 double-positive cells were present at relatively low frequencies, our results are consistent with recently published data showing that rhesus macaques who were protected from developing TB following mucosal vaccination with BCG harbored mycobacteria-specific IFN- $\gamma / \mathrm{IL}-17$ double-positive $\mathrm{CD}^{+} \mathrm{T}$ cells (16). Together, the data represented in Figure 5, Figure 6, and Supplemental Figure 3, A-D, show that in addition to $M$. tuberculosis antigen-specific IFN- $\gamma^{+}$T cells, $M$. tuberculosis-specific IL-17 ${ }^{+}$and IFN- $\gamma /$ IL-17 double-positive T cells are present in the airways of asymptomatic rhesus macaques, suggesting an association between balanced Th1/Th17 responses at mucosal sites of infection and immune control of $M$. tuberculosis infection.

M. tuberculosis-specific IFN- $\gamma^{+}$and $I L-17^{+} C D 4^{+} T$ cells in the airways coexpress $C X C R 3$ and CCR6. Chemokine receptors CXCR3 and CCR6 regulate the migration of antigen-specific Th1 and Th17 cells, respectively, 
A
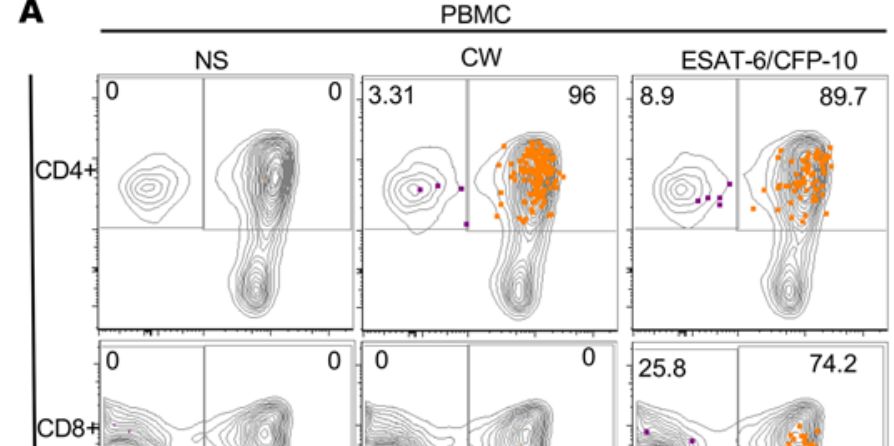

เั

C

CW

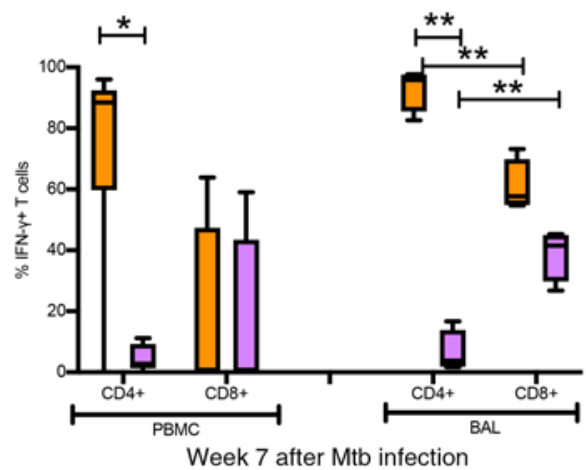

E

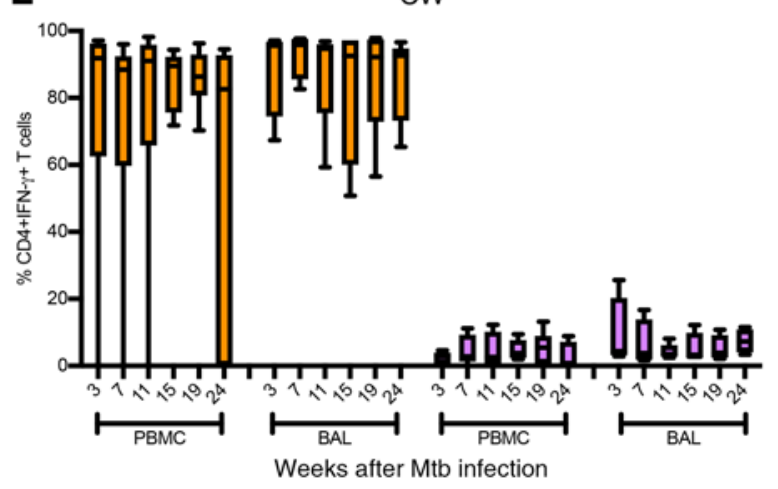

G

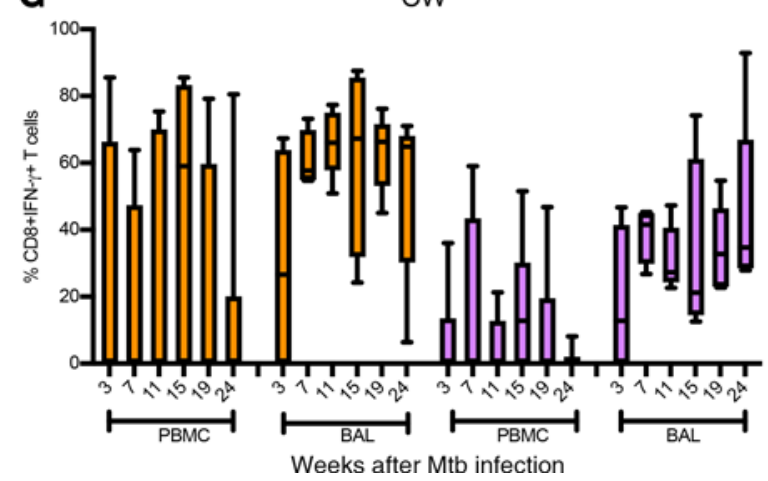

D

H
B

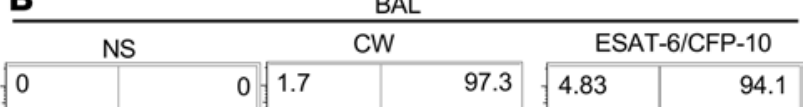

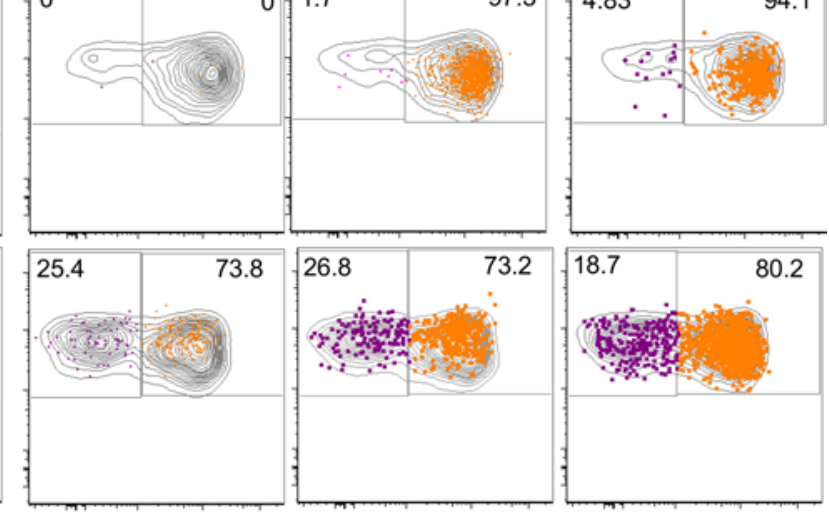

Week 7 after Mtb infection

$\mathrm{CD} 28+\mathrm{CD} 95+$

ESAT-6/CFP-10
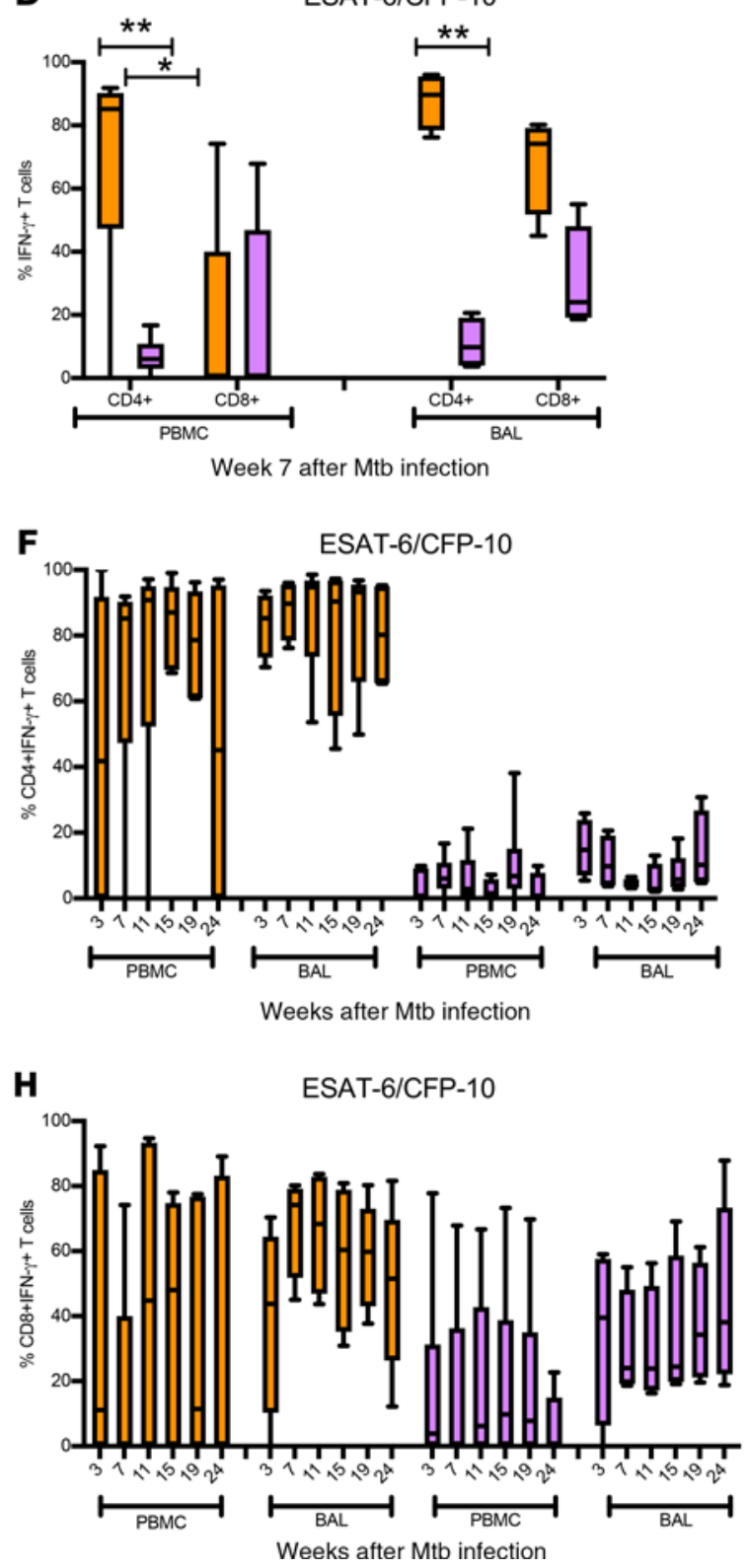
Figure 4. Memory phenotypes of $M$. tuberculosis-specific CD4 $4^{+}$and $\mathbf{C D 8} 8^{+}$T cells in PBMCs and BAL. (A and B) Representative flow plots of IFN- $\gamma^{+}$CD4 $4^{+}$and CD8 ${ }^{+}$T cells expressing CD28 and CD95 in PBMCs and in BAL, respectively, following CW and ESAT-6/CFP-10 stimulation or no stimulation (NS). IFN- $\gamma$-producing $\mathrm{CD}^{+} / \mathrm{CD}^{+}$cells were overlaid on $\mathrm{CD} 4^{+} / \mathrm{CD}^{+}$cells expressing $\mathrm{CD28} 8^{+}$and $\mathrm{CD95} 5^{+}$. Antigen-specific memory (CD28+CD95 $5^{+}$and effector cells (CD28-CD95 $5^{+}$were measured by gating first on $\mathrm{CD} 4^{+}$and $\mathrm{CD} 8^{+} \mathrm{T}$ cells, followed by gating on IFN- $\gamma^{+} \mathrm{CD} 4^{+}$or CD8 $8^{+} \mathrm{T}$ cells, then gated for CD28 and CD95. (C and D) Frequencies of CWand ESAT-6/CFP-10-specific memory (CD28+CD95+, orange bar) and effector (CD28-CD95+, purple bar) CD4+ and CD8 ${ }^{+}$cells in PBMCs and BAL at week 7 post-M tuberculosis infection of macaques $(n=6)$ with LTBI. (E-H) Kinetics of CW- and ESAT-6/CFP-10-specific memory (orange bar) and effector (purple bar) CD4+ and CD8 ${ }^{+} \mathrm{T}$ cells in PBMCs and BAL. Median line, 25th and 75th percentiles (boundaries of boxes), and 5 th and 95th percentiles (whiskers above and below box plots) are indicated in the box plots. Two-way ANOVA with Holm-Šidák multiple corrections test was used to calculate the statistical difference. ${ }^{* *} P<0.01,{ }^{*} P<0.05$.

into inflamed mucosal tissues following microbial infection (47-49). We therefore sought to determine whether these chemokine receptors were expressed on the $M$. tuberculosis-specific IFN- $\gamma^{+}$and IL- $17^{+} \mathrm{CD} 4^{+}$ $\mathrm{T}$ cells present in the airways of macaques that controlled $M$. tuberculosis infection. We assessed the frequencies of CW- and ESAT-6/CFP-10-specific IFN- $\gamma^{+}$and IL-17 ${ }^{+} \mathrm{CD} 4^{+} \mathrm{T}$ cells in the BAL that expressed either CXCR3 or CCR6 (Figure 7, A and B) and found that the majority of IFN- $\gamma$ - and IL-17-producing CD4 ${ }^{+}$ $\mathrm{T}$ cells expressed CXCR3 (mean \pm SEM at week 7 post $-M$. tuberculosis infection, IFN- $\gamma^{+} 82.4 \% \pm 2.9 \%$, IL- $17^{+} 61 \% \pm 7 \%$ ) and CCR6 (mean \pm SEM at week 7 post $M$. tuberculosis infection, IFN- $\gamma^{+} 56 \% \pm 8.6 \%$, $\mathrm{IL}-17^{+} 67 \% \pm 11 \%$ ). To assess coexpression of CXCR3 and CCR6, we analyzed samples from the week 7 time point because the low frequencies of IL-17-producing $\mathrm{T}$ cells at later time points precluded reliable analyses. We found that the majority of $M$. tuberculosis-specific IFN- $\gamma^{+}$(Figure 7, C and D) and IL-17 ${ }^{+}$(Figure 7, $\mathrm{E}$ and $\mathrm{F}$ ) $\mathrm{CD}^{+} \mathrm{T}$ cells in BAL coexpressed CXCR3 and CCR6. In addition, IFN- $\gamma$-producing CD4 ${ }^{+}$ cells coexpressing CCR6 and CXCR3 were also the major subset in BAL at later time points (Supplemental Figure 4, A and B). Moreover, IFN- $\gamma / \mathrm{IL}-17$ double-positive cells were also predominantly $\mathrm{CXCR} 3^{+} \mathrm{CCR} 6^{+}$ (Figure 7, G and H). Overall, CD4 ${ }^{+} \mathrm{T}$ cells coexpressing CXCR3 and CCR6 were the main IFN- $\gamma-$ and IL-17-producing subsets present in the airways of macaques with LTBI.

$C X C R 3^{+} C D 4^{+} T$ cells in the lung correlate with lung $M$. tuberculosis burden. Our observation that CXCR3 and CCR6 coexpressing cells were the predominant $M$. tuberculosis antigen-specific CD4 ${ }^{+} \mathrm{T}$ cells in the airways of macaques with LTBI prompted us to investigate the localization and frequencies of these cells in the lungs of $M$. tuberculosis-infected NHPs by immunostaining of lung tissue sections. While $\mathrm{CXCR}^{+}{ }^{+} \mathrm{CD} 4^{+} \mathrm{T}$ cells were clearly detected in lung tissue, robust immunostaining for CCR6 in paraffin-fixed lung tissue could not be established, despite extensive efforts. Because most of the CCR6 ${ }^{+} \mathrm{CD} 4^{+}$ $\mathrm{T}$ cells in BAL were also positive for CXCR3 (Figure 7), we used CXCR3 as a proxy for both chemokine receptors (Figure 8). We also stained lung sections with antibodies against CD163 and CD68 to identify macrophages (50). Immunostaining of lung sections from animals with asymptomatic LTBI showed that $\mathrm{CXCR}^{+} \mathrm{CD}^{+} \mathrm{T}$ cells were present in lung tissue (Figure $8 \mathrm{~A}$ ), with higher densities of $\mathrm{CXCR}^{+} \mathrm{CD}^{+} \mathrm{T}$ cells in granulomatous areas of the lung compared with nongranulomatous areas (Figure 8B). Next, to investigate the relationship between lung $\mathrm{CD}^{+} \mathrm{CXCR} 3^{+}$cells and $M$. tuberculosis burden, we stained archived lung tissue sections from rhesus macaques with active TB disease (42). Quantification of the density of $\mathrm{CXCR} 3^{+} \mathrm{CD} 4^{+}$cells in the lungs of animals with active $\mathrm{TB}$ showed that the density of $\mathrm{CXCR} 3^{+} \mathrm{CD} 4^{+}$cells was significantly $(P=0.03)$ higher in the granulomatous areas compared with nongranulomatous areas of the lung, with overall lower $\mathrm{CXCR} 3^{+} \mathrm{CD} 4{ }^{+}$densities in the lungs of active TB relative to LTBI (Figure 8B). Interestingly, we observed a negative correlation between lung $\mathrm{CXCR}^{+} \mathrm{CD}^{+}$densities and lung CFU (Figure $8 \mathrm{C}$ ), suggesting that $\mathrm{CXCR} 3^{+} \mathrm{CD} 4{ }^{+} \mathrm{T}$ cells are associated with lower $M$. tuberculosis burdens. These results, along with the results reported in Figure 6 and Figure 7, suggest that recruitment of CXCR3- and CCR6-expressing Th1 and Th17 subsets to the airways and lungs of macaques likely contributes to immune control of M. tuberculosis burden.

\section{Discussion}

Asymptomatic IGRA ${ }^{+}$individuals with LTBI can remain disease free for decades, but current tests cannot determine whether they harbor bacteria or have cleared infection. Thus, defining immune responses associated with establishing and maintaining human LTBI remains challenging. Macaque models recapitulate multiple aspects of human $M$. tuberculosis infection and disease progression. Both cynomolgus and rhesus macaque species are attractive animal models for studying LTBI (35-42, 51-55). Low-dose infection with $M$. tuberculosis CDC1551 has been shown to lead to asymptomatic infection in $80 \%$ of rhesus macaques with approximately $20 \%$ progressing to active TB disease (38-42). In cynomolgus macaques, approximately $50 \%$ of animals infected with a low dose of $M$. tuberculosis Erdman develop asymptomatic, latent TB, with the rest developing 

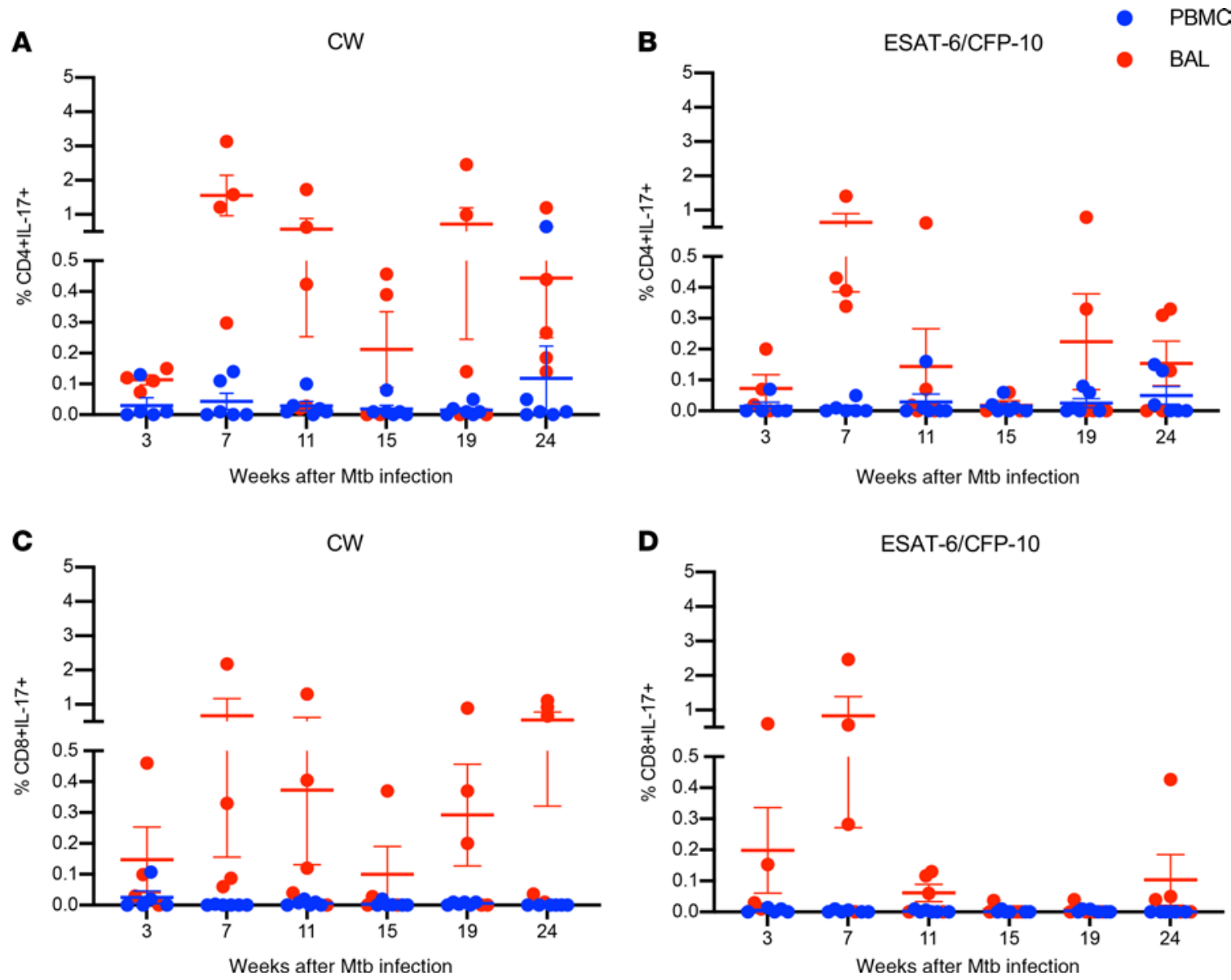

Figure 5. Kinetics of $\mathbf{M}$. tuberculosis-specific CD4 $^{+}$and CD8 ${ }^{+}$T cells producing IL-17 in PBMCs and BAL. PBMC (blue circles) and BAL (red circles) samples $(n=6)$ were stimulated with CW $(\mathbf{A}$ and $\mathbf{C})$ and ESAT-6/CFP-10 peptide pools $(\mathbf{B}$ and $\mathbf{D})$, and IL-17 production by CD4 ${ }^{+}(\mathbf{A}$ and $\mathbf{B})$ and CD8 ${ }^{+}(\mathbf{C}$ and $\mathbf{D})$ T cells was assessed by ICS and flow cytometry at the time points indicated (weeks) post-M. tuberculosis infection. Wilcoxon matched-pairs signed rank test was used to compare the frequencies of IL-17-producing $C D 4^{+}$and $C D 8^{+}$T cells between BAL and PBMCs. Horizontal lines indicate the mean with SEM.

active TB $(37,52,56-59)$. Differences in disease progression between these 2 species and M. tuberculosis strain combinations enables study of different aspects of human M. tuberculosis infection and disease. We have previously shown that rhesus macaques infected with low-dose CDC1551 develop asymptomatic $M$. tuberculosis infection and harbor viable $M$. tuberculosis bacteria in their lungs for up to 9 months (39). Moreover, coinfection with simian immunodeficiency virus (SIV) induced reactivation to TB disease while treatment with isoniazid and rifapentine for 3 months prevented SIV-mediated reactivation to TB. Therefore, to characterize immune responses associated with LTBI, we used our previously established low-dose rhesus macaque model of asymptomatic LTBI to study the nature and kinetics of M. tuberculosis antigen-specific T cells for up to 24 weeks postinfection. We observed varying degrees of lung inflammation and granulomas across all the asymptomatic animals (Figure 1E and Figure 8), consistent with the heterogeneity reported in cynomolgus macaques with LTBI, where both sterile and nonsterile granulomas were present within a single host (60).

Previous studies in NHPs assessed antigen-specific immune responses in BAL at early time points in infected macaques $(52,61-63)$ or during vaccination $(16,64-66)$. However, in-depth analysis of the phenotypes, functionality, and kinetics of M. tuberculosis antigen-specific immune responses in blood and BAL during establishment and maintenance of asymptomatic latent infection has been lacking. To our knowledge, our current study, in which we undertook monthly collection of blood and BAL samples starting at week 3 postinfection and up to 24 weeks, is the first to longitudinally profile $M$. tuberculosis-specific (CW- and ESAT-6/CFP-10-specific) $\mathrm{CD} 4^{+}$and $\mathrm{CD} 8^{+} \mathrm{T}$ cell responses during LTBI and to directly compare peripheral blood and lung compartments over an extended period of asymptomatic infection. We found that the frequencies of M. tuberculosis CW- and ESAT-6/CFP-10-specific IFN- $\gamma$-producing $\mathrm{CD} 4^{+}$and $\mathrm{CD} 8^{+} \mathrm{T}$ cell responses were significantly higher in BAL (51-fold for $\mathrm{CD} 4$ 
A

PBMC

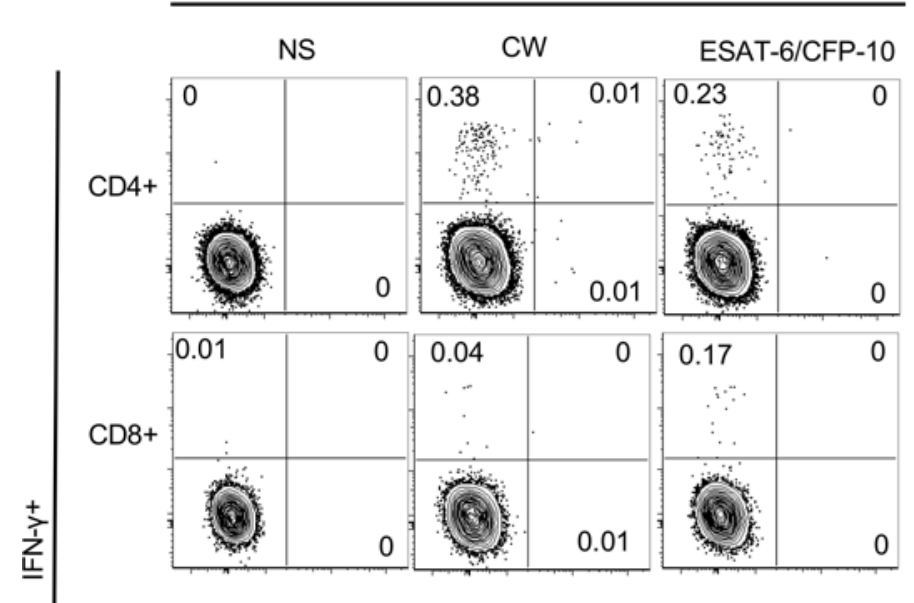

BAL
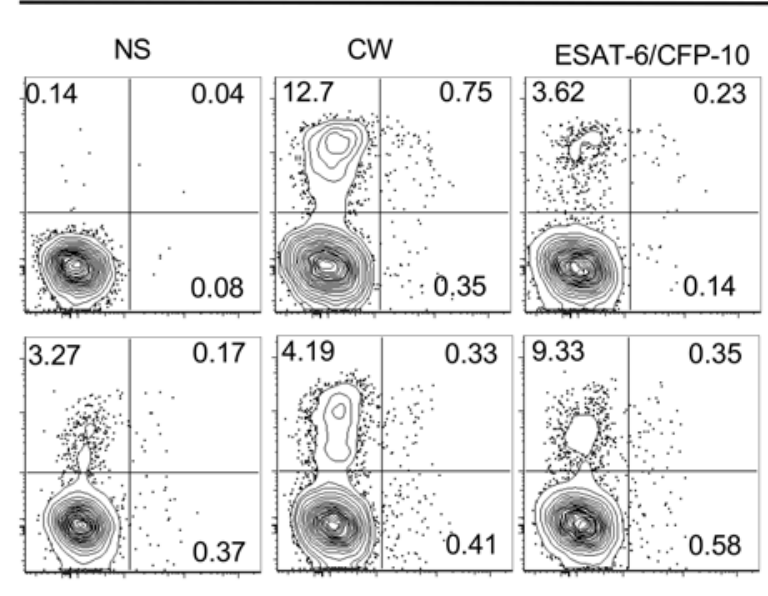

B

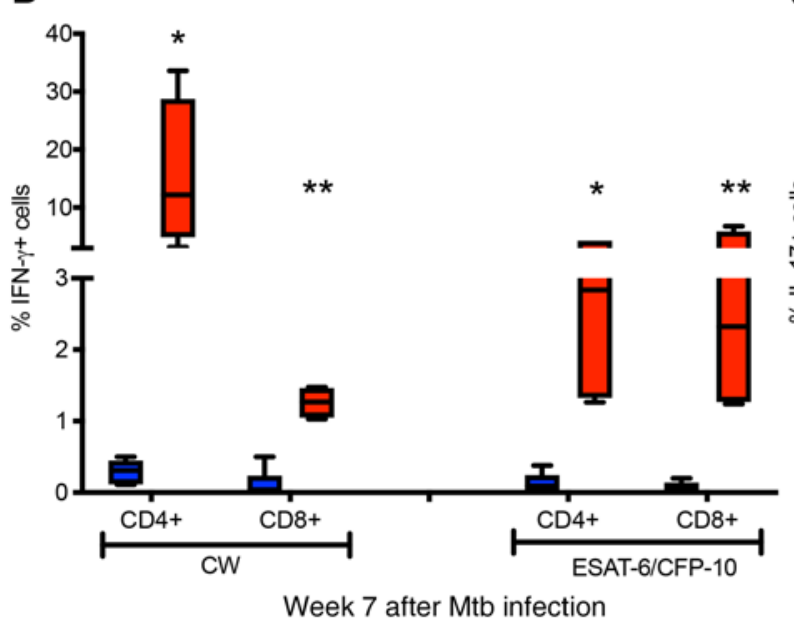

D

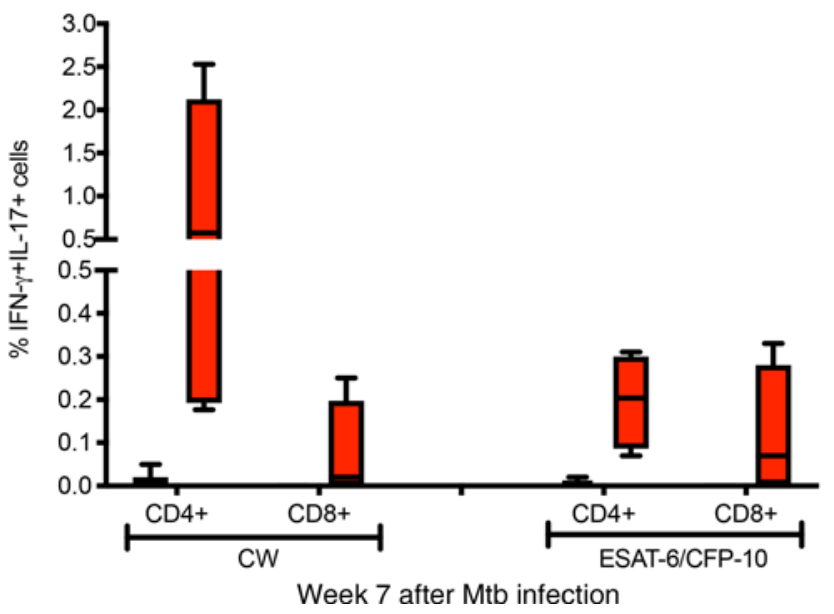

C

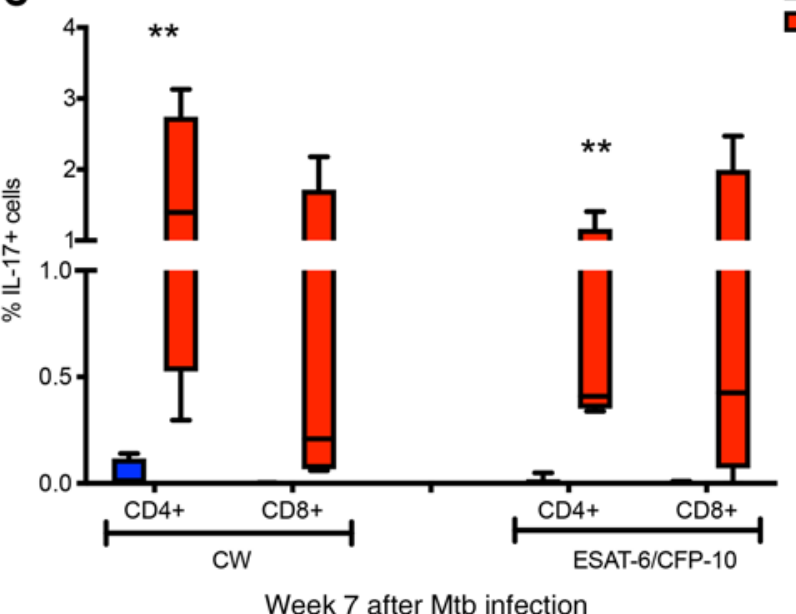

Figure 6. Higher frequencies of IL-17+ and IFN- $\gamma^{+} \mathrm{IL}-17^{+} M$. tuberculosisspecific $\mathrm{CD4}^{+} \mathrm{T}$ cells in the BAL compared with PBMCs. (A) Representative flow plots of week 7 PBMCs and BAL that are either nonstimulated (NS) or stimulated with CW and ESAT-6/CFP-10 peptide pools. CD4 ${ }^{+}$and CD8 ${ }^{+}$ T cells expressing IFN- $\gamma$ and IL-17 were assessed by ICS and flow cytometry. Frequencies of single-positive IFN- $\gamma$ (B), single-positive IL-17 (C), and IFN- $\gamma /$ IL-17 double-positive (D) CD4 $4^{+}$and $C D 8^{+} T$ cells in PBMCs (blue) and BAL (red) from macaques $(n=6)$ with LTBI. Median line, 25th and 75th percentiles (boundaries of boxes), and 5th and 95th percentiles (whiskers above and below box plots) are indicated in the box plots. Two-way ANOVA with Holm-Šidák multiple corrections test was used to calculate the statistical difference. ${ }^{* *} P<0.01,{ }^{*} P<0.05$.

and 30-fold for CD8; Figure 3) compared with PBMCs, with higher proportions of $\mathrm{CD} 8^{+} \mathrm{T}$ cells present in BAL compared with their $\mathrm{CD} 4^{+} \mathrm{T}$ cell counterparts. The presence of high frequencies of M. tuberculosis-specific T cells in the airways as early as 3 weeks after $M$. tuberculosis infection, and their persistence throughout the study until 24 weeks, suggests that accumulation of $M$. tuberculosis-specific T cells at mucosal sites during LTBI likely contributes to control of $M$. tuberculosis infection. 
A

ESAT-6/CFP-10

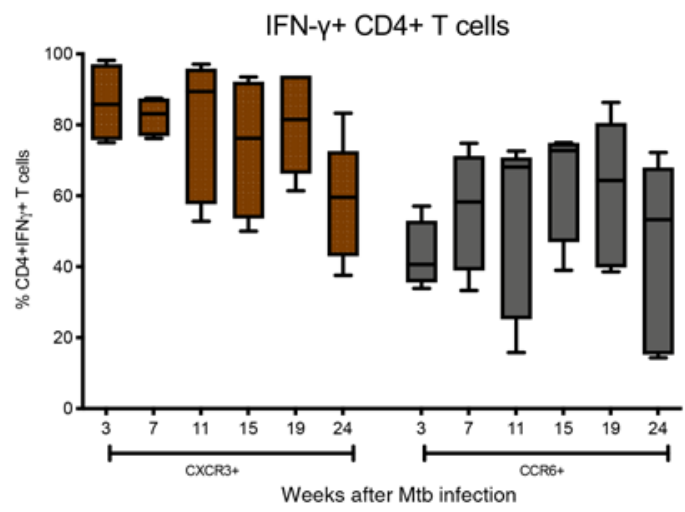

C

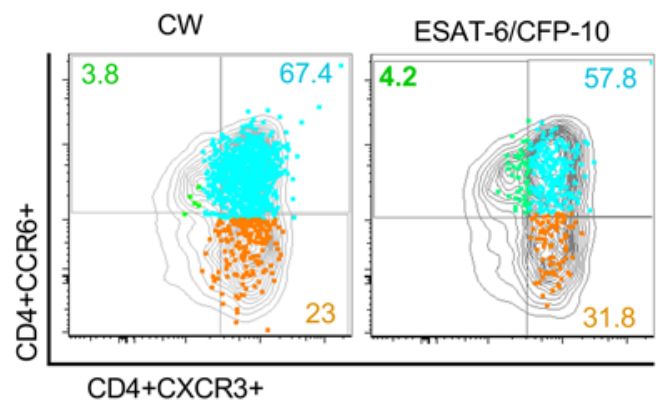

E

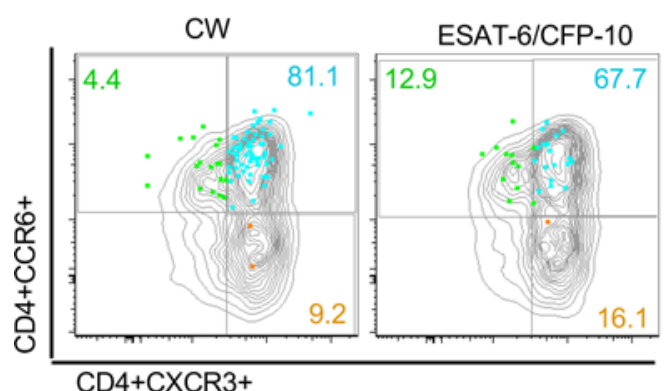

G

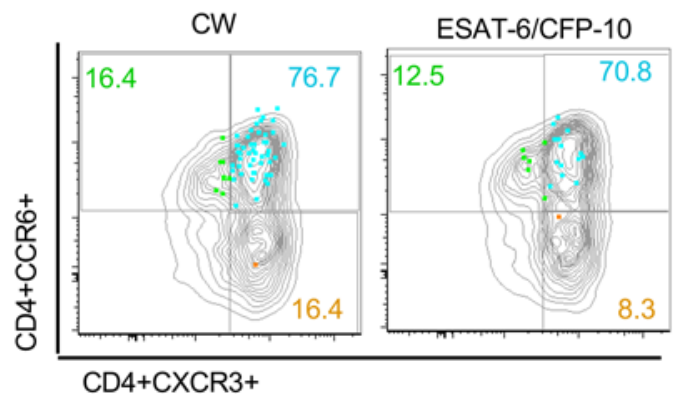

B

ESAT-6/CFP-10

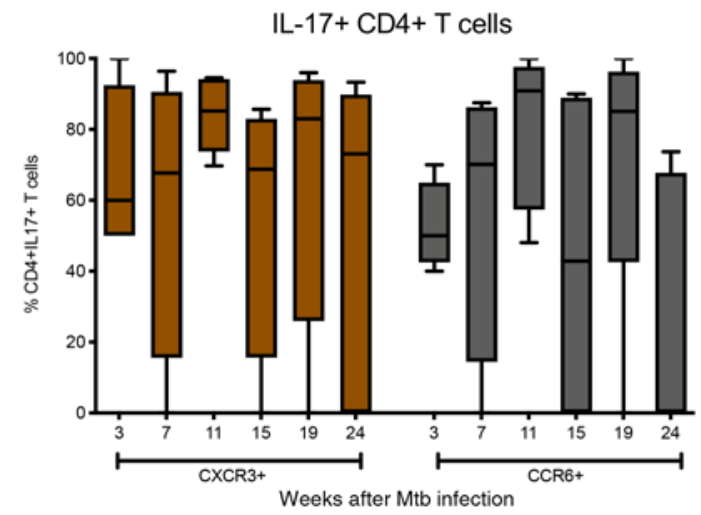

D

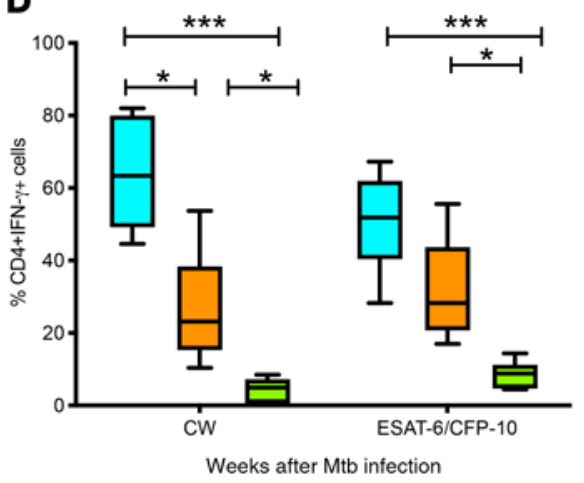

$\mathbf{F}$

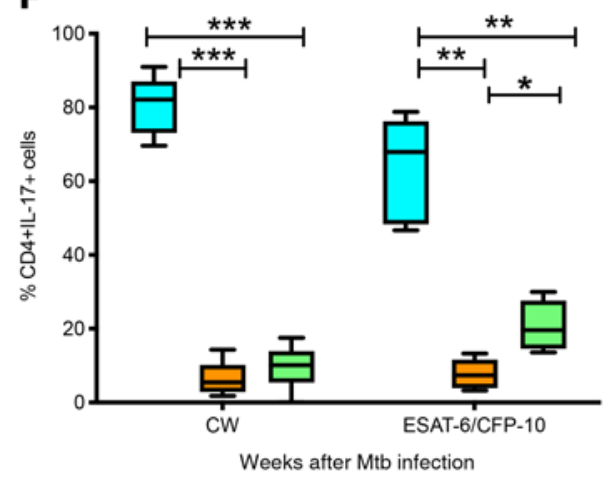

H

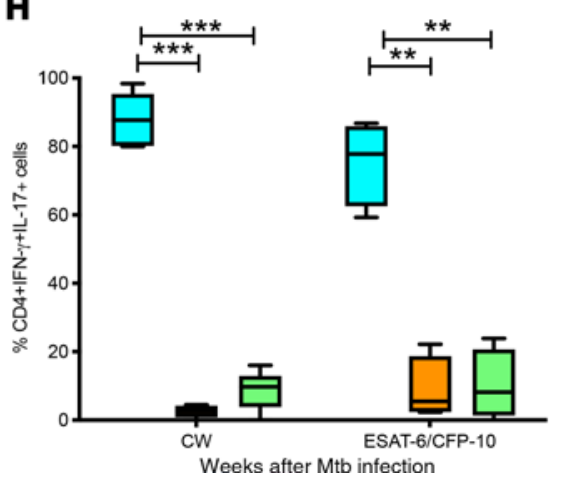

CXCR3+CCR6+

CXCR3+CCR6-

CXCR3-CCR6+

Figure 7. M. tuberculosisspecific $\mathrm{CD4}^{+} \mathrm{T}$ cells producing IFN- $\gamma$ and IL-17 coexpressed CXCR3 and CCR6. Frequencies of ESAT-6/CFP-10-specific IFN- $\gamma^{+}(\mathbf{A})$ and IL-17+ (B) CD4+ T cells in the BAL $(n=6)$ that expressed either CXCR3 (brown bar) or CCR6 (black bar). To measure antigen-specific CCR6 and $\mathrm{CXCR} 3^{+}$cells, we first gated on $\mathrm{CD}^{+}$and $\mathrm{CD} 8^{+} \mathrm{T}$ cells, followed by IFN $-\gamma^{+} \mathrm{CD} 4^{+}$and $\mathrm{CD} 8^{+}$ T cells, then gated for CCR6 and CXCR3. Majority of IFN- $\gamma$ - and IL-17-producing CD4 ${ }^{+} T$ cells expressed CXCR3 at all time points. M. tuberculosis-specific IFN- $\gamma^{+}(\mathbf{C}$ and $\mathbf{D})$ and $\mathrm{IL}-17^{+}$(E and F) $C D 4^{+} T$ cells in BAL coexpressing CXCR3 and CCR6 (blue bar) were significantly higher compared with $\mathrm{CXCR3}^{+} \mathrm{CCR6}^{-}$ (orange bar) and CXCR3-CCR6 ${ }^{+}$ (green bar) subsets. IFN- $\gamma^{+}$I IL-17+ cells were also predominantly CXCR3 ${ }^{+} \mathrm{CCRG}^{+}$(G and $\left.\mathbf{H}\right)$. Median line, 25th and 75th percentiles (boundaries of boxes), and 5th and 95th percentiles (whiskers above and below box plots) are indicated in the box plots. Two-way ANOVA with Holm-Šidák multiple corrections test was used to calculate the statistical difference. ${ }^{* * *} P<$ $0.001,{ }^{*} P<0.01,{ }^{*} P<0.05$ 
A $\mathrm{CD} 163 / \mathrm{CD} 68 / \mathrm{CD} 4 / \mathrm{CXCR} 3$
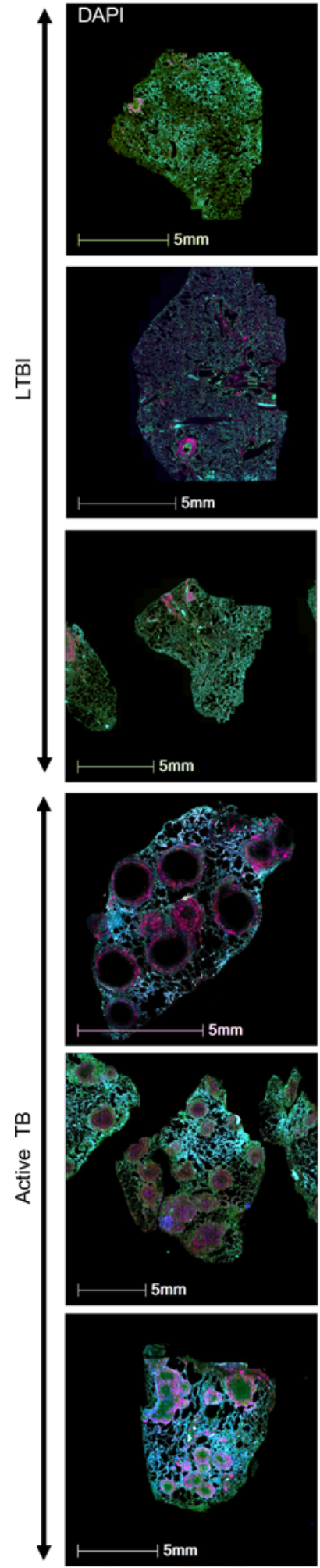

Granuloma
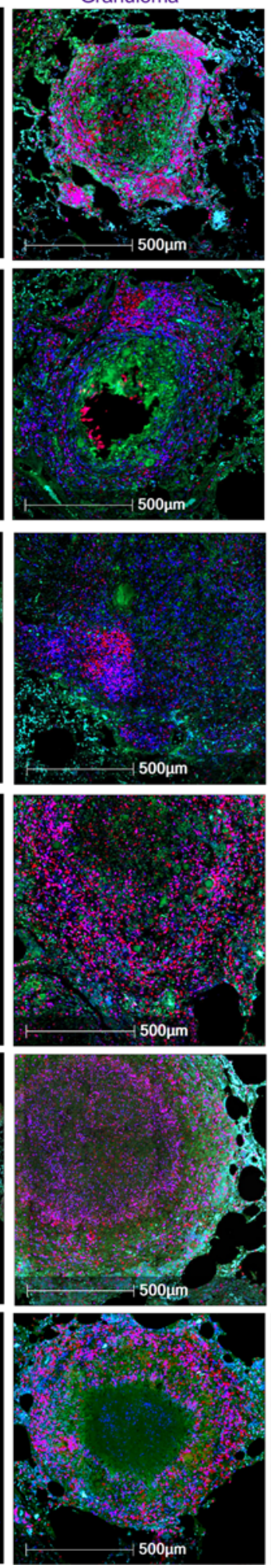

Non-granuloma
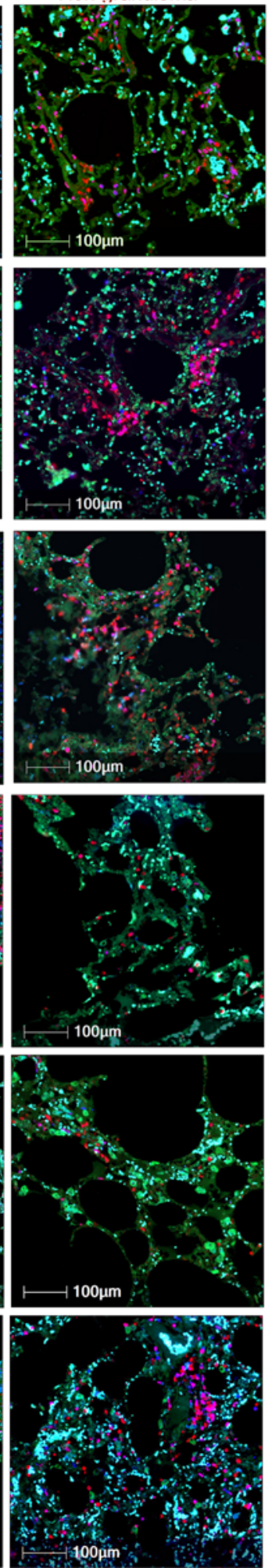

B
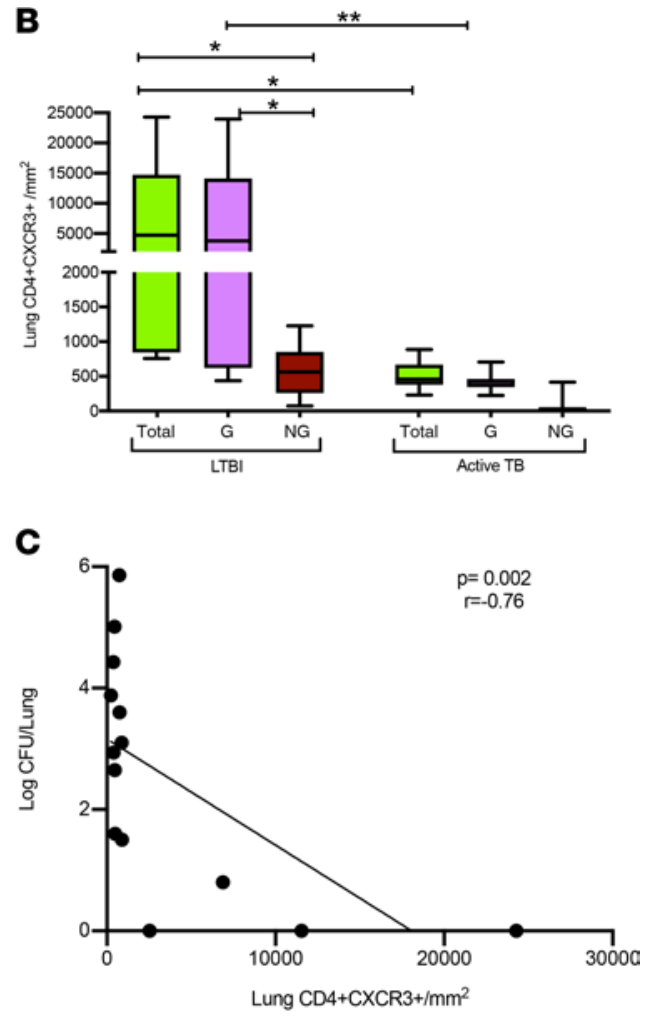

Figure 8. CXCR3 ${ }^{+} \mathrm{CD} 4^{+} \mathrm{T}$ cells were predominant in the granulomatous region of the lung. (A) Representative immunohistochemistry staining of lung sections from macaques with LTBI and active TB. Macrophage (green), CD4 (blue), CXCR3 (red), and nuclei (DAPI). Left panel shows low-magnification $(5 \mathrm{~mm}$ ) images of lung sections from macaques with asymptomatic LTBI and active TB. Middle and right panels show images of the granulomatous $(500 \mu \mathrm{m})$ and nongranulomatous (100 $\mu \mathrm{m})$ areas of lung sections, respectively. (B) Densities of $\mathrm{CD}^{+}{ }^{+} \mathrm{CXCR3^{+ }}$ cells (purple) in lung tissue of animals with LTBI $(n=6)$ and active TB $(n=8)$ in the lung (total) and in granulomatous (C) and nongranulomatous (NG) areas of the lung. Density of $\mathrm{CD4} 4^{+} \mathrm{CXCR} 3^{+}$cells was measured by dividing the number of $\mathrm{CD} 4^{+} \mathrm{CXCR} 3^{+}$cells quantified by the area (in $\mathrm{mm}^{2}$ ) calculated from H\&Estained tissue using algorithms trained via a deep convolutional network (HALO, Indica Labs). Median line, 25th and 75th percentiles (boundaries of boxes), and 5th and 95th percentiles (whiskers above and below box plots) are indicated in the box plots. Two-way ANOVA with Holm-Šidák multiple corrections test was used for the statistical analysis. ${ }^{*} P<0.05$. (C) Lung bacterial burden negatively $(P=0.002)$ correlated with the density of lung CXCR3 ${ }^{+} C D 4^{+} T$ cells from animals with LTBI $(n=6)$ and active TB $(n=8)$. Correlation was performed using nonparametric Spearman correlation method.

Interestingly, in addition to antigen-specific IFN- $\gamma^{+} \mathrm{CD}^{+} \mathrm{T}$ cells, we also detected $M$. tuberculosisspecific IL- $17^{+} \mathrm{CD} 4^{+}$and $\mathrm{CD} 8^{+} \mathrm{T}$ cells in the BAL by 3 weeks post $-M$. tuberculosis infection. $M$. tuberculosis-specific IL-17 ${ }^{+} \mathrm{T}$ cells peaked at week 7 and were detected mainly in BAL, being largely absent in blood. The accumulation of IL-17 cytokine-producing CD4 $4^{+} \mathrm{T}$ cells in lung compartments is consistent with the known preferential accumulation of Th17 cells at mucosal sites during infection (67). Various 
studies in mice have also suggested that Th17 cells play an important role in protective immunity against $\mathrm{TB}$, both in the context of vaccination (68) as well as in the context of disease progression $(13,69)$. In addition, studies in macaques with active TB disease have suggested an association between granuloma IL- $17^{+}$cells and upregulation of genes related to Th17 cells and control of $M$. tuberculosis infection (14, 70). Interestingly, in addition to antigen-specific IL- $17^{+} \mathrm{CD} 4^{+} \mathrm{T}$ cells, we also observed IL- $17^{+} \mathrm{CD} 8^{+} \mathrm{T}$ cells, as well as IFN- $\gamma^{+} \mathrm{IL}-17^{+} \mathrm{CD} 4^{+} \mathrm{T}$ cells in the BAL. Our results indicate that both IFN- $\gamma^{+}$and IFN- $\gamma^{+} \mathrm{IL}-17^{+}$ cells emerge soon after $M$. tuberculosis infection and persist for several weeks, thus likely contributing to controlling $M$. tuberculosis infection during LTBI. While frequencies of antigen-specific IL- $17^{+}$and IFN- $\gamma /$ IL-17 double-positive CD4 ${ }^{+} \mathrm{T}$ cells were relatively low, these data support the idea that balanced Th1 and Th17 responses are associated with enhanced immunity to TB. Future studies that selectively deplete IL-17- or IFN- $\gamma$-producing CD4 ${ }^{+} \mathrm{T}$ cells, or other IL-17-producing cell types such as CD ${ }^{+} \mathrm{T}$ cells, $\gamma \delta \mathrm{T}$ cells, mucosal-associated invariant T cells, or innate lymphoid cells, in the context of LTBI or vaccination in macaques, will provide more direct evidence for the role of IL-17 and Th17 cells in controlling $M$. tuberculosis infection. Moreover, determining the balance between Th1/Th17 responses in animals with active TB at early time points postinfection and studying the potential pathogenic effects of IL-17 during chronic infection will shed light on protective versus pathogenic aspects of IL-17 function in TB.

Elicitation and maintenance of memory responses has been associated with protective immunity (44, 45). In the present study, M. tuberculosis antigen-specific memory $\mathrm{CD} 4^{+}$and $\mathrm{CD} 8^{+} \mathrm{T}$ cells, both in $\mathrm{PBMCs}$ and in BAL, were maintained at high levels throughout the time course of the study, suggesting that these cells are likely to be long-lived and involved in maintenance of the asymptomatic LTBI state. Although $\mathrm{CD}^{+} \mathrm{T}$ cells were the predominant subset responding to $M$. tuberculosis infection, we also detected antigen-specific IFN- $\gamma^{+} \mathrm{CD} 8^{+} \mathrm{T}$ cells, which increased in frequency between 3 and 7 weeks in parallel with antigen-specific $\mathrm{CD}^{+} \mathrm{T}$ cells. The $\mathrm{CD}^{+} \mathrm{T}$ cell responses that we observed in macaques that control infection differ from previous mouse studies in which lung $\mathrm{CD} 8^{+} \mathrm{T}$ cell responses were considerably delayed compared with $\mathrm{CD}^{+} \mathrm{T}$ cell responses (71). Additionally, our study shows that $M$. tuberculosis-specific CD8 ${ }^{+}$ T cells producing IL-17 are present, although coproduction of IFN- $\gamma$ and IL-17 was reduced relative to the $\mathrm{CD}^{+}$subsets. Overall, our findings show that $M$. tuberculosis-specific T cells produce both IFN- $\gamma$ and IL-17 in the BAL at higher frequencies compared with blood and suggest that these responses may be associated with control of $M$. tuberculosis during asymptomatic $M$. tuberculosis infection.

Th1 and Th17 cells can be identified by the expression of chemokine receptors; specifically CXCR 3 and CCR6 are considered surface marker for Th1 and Th17, respectively (72). Within the CXCR3 and CCR6 axis, another subset of cells that coexpress both CXCR3 and CCR6 have been identified (73,) and these cells produce both cytokines IFN- $\gamma$ and IL-17. These IFN- $\gamma / \mathrm{IL}-17$ double-positive CD4 ${ }^{+} \mathrm{T}$ cells have been shown to play a pathogenic role during autoimmune diseases (74), but their role in protective immunity to infection remains unclear. With respect to $M$. tuberculosis infection, a recent study showed preferential expansion of $\mathrm{CXCR}^{+}{ }^{+} \mathrm{CCR} 6^{-}$and reduction in $\mathrm{CXCR} 3^{-} \mathrm{CCR} 6^{+} \mathrm{CD}^{+}$subsets in individuals with $\mathrm{TB}$ immune reconstitution inflammatory syndrome (75). In latently infected individuals, $\mathrm{CD}^{+} \mathrm{T}$ cells that mainly coexpress CXCR3 and CCR6 that produced IFN- $\gamma$ were reported to be present in peripheral blood, but these cells did not express IL-17 $(73,76,77)$. However, the coexpression pattern of CXCR3 and CCR6 on M. tuberculosisspecific $\mathrm{T}$ cells present in BAL during latent infection has not been studied to our knowledge. In the current study, we found that the majority of antigen-specific CD4 ${ }^{+} \mathrm{T}$ cells coexpressed CXCR3 and CCR6 in BAL (Figure 7), and interestingly, unlike in blood, where IL-17-producing cells were absent (Figure 5 and Figure 6), $\mathrm{CXCR}^{+} \mathrm{CCR} 6^{+}$subsets in macaque BAL were $\mathrm{IL}-17^{+}$and $\mathrm{IFN}-\gamma^{+} \mathrm{IL} 17^{+}$coproducing cells.

In $M$. tuberculosis-infected macaques, CXCR3 ${ }^{+}$Th1 cells have been shown to be efficient in localizing to lung parenchyma during active TB (61), and in mouse models, these cells have been implicated in containing salmonella within granulomas (78). However, the location of $\mathrm{CXCR} 3^{+} \mathrm{CD} 4^{+}$cells in the lung and their association with $M$. tuberculosis control in rhesus macaque lungs during LTBI have not been previously described to our knowledge. Using immunofluorescence staining and quantification of $\mathrm{CXCR} 3^{+} \mathrm{CD} 4^{+}$cells in lung tissue sections from animals with asymptomatic LTBI and active TB, we found that $\mathrm{CXCR} 3^{+} \mathrm{CD} 4^{+}$cells were located largely in granulomatous areas. Importantly, we found that the density of $\mathrm{CXCR} 3^{+} \mathrm{CD} 4^{+}$cells in granulomas correlated inversely with $M$. tuberculosis load (Figure 8), suggesting that these cells may have protective functions. Although we were unable to effectively stain for CCR6 in the lung, because the majority of $\mathrm{CCR}^{+}$cells in the BAL also expressed CXCR3 (Figure 7 and Supplemental Figure 4), we hypothesize that $\mathrm{CXCR} 3^{+} \mathrm{CCR} 6^{+} \mathrm{T}$ cells subsets are likely to be associated with mycobacterial control in the lung. Our 
studies have some limitations: while we have shown a negative correlation between $\mathrm{CXCR} 3^{+} \mathrm{CD} 4^{+} \mathrm{T}$ cells in the lung and $M$. tuberculosis burden, we have not established causality or the mechanistic basis for CXCR $3^{+} \mathrm{C}-$ $\mathrm{CR}^{+}$Th1/Th17 responses in controlling $M$. tuberculosis. Moreover, we were unable to longitudinally compare the kinetics of $\mathrm{CXCR} 3^{+} \mathrm{CCR} 6^{+} \mathrm{Th} 1$ and $\mathrm{Th} 17$ responses in lung compartments of animals with active TB because we did not have sufficient numbers of animals that progressed to TB in this study. However, we anticipate extending our studies to investigating $M$. tuberculosis antigen-specific immune responses associated with TB progression versus immune control by leveraging stored samples from animals that developed active $\mathrm{TB}$, from this study as well as from different ongoing and future studies.

In summary, our studies clearly show that lung compartments of rhesus macaques that control $M$. tuberculosis infection are populated with high levels of M. tuberculosis-specific T cells producing IFN- $\gamma$ and IL-17. These cells coexpress CXCR3 and CCR6, and their presence correlates with lowered M. tuberculosis burdens in the lung. Our study also highlights the unique value of the macaque model of LTBI for studying correlates of protective immunity to $\mathrm{TB}$ at mucosal sites of infection. Future studies include understanding the crosstalk between innate immune cells and development of $M$. tuberculosis-specific $\mathrm{T}$ cell responses in the BAL and lungs of animals that progress to TB versus those that remain asymptomatic. In addition, perturbing the latent state by depleting specific immune cell subsets or by using immunosuppressive agents, such as SIV, will further extend our understanding of protective immunity to TB and advance the development of better vaccines and immune therapeutics.

\section{Methods}

Infection of animals with $M$. tuberculosis. The experimental design of these studies is described in Figure 1. Eight Indian-origin adult rhesus macaques (Macaca mulatta) were exposed to approximately 10 CFU of $M$. tuberculosis CDC1551 as described previously (38), resulting in all animals being infected, as assessed by the development of positive TSTs and IGRAs. The animals were obtained from the Tulane National Primate Research Center (TNPRC) breeding colony. Before the study, the animals were quarantined for 90 days and tested by both TST and an NHP-specific IGRA (PRIMAGAM, Prionics) (38) to ensure they were not previously exposed to $M$. tuberculosis infection. A custom head-only dynamic inhalation system housed within a class III biological safety cabinet was used for this purpose $(38,42,45,79)$. All animals tested positive by PRIMAGAM and TST at 3 and 7 weeks postinfection. Six out of 8 animals did not exhibit any signs or symptoms of active TB and were considered to have LTBI when they remained asymptomatic for 15 weeks. All 6 animals remained asymptomatic for the duration of the 24-week study. Two out of 8 animals developed progressive primary TB disease and exhibited pyrexia, wasting, high serum CRP, and other clinical signs of TB by 7 weeks post- $M$. tuberculosis infection and were included in a different study. M. tuberculosis-infected animals were housed under Biological Safety Level 3 conditions.

Clinical procedures and sample collection. Procedures for weekly complete blood counts, chemistry, CXRs, and BAL at week 3, every 4 weeks thereafter, and necropsy (week 24) have been described previously (38, 42, 50, 80). CXRs were scored by veterinary clinicians in a blinded fashion on a subjective scale of $0-4$, with a score of 0 denoting normal lung and a score of 4 denoting severe tuberculous pneumonia, as previously described (38). Measurements of CRP, body weight, and temperature were performed as described earlier (38), at week -1 and at weeks 3, 7, 11, 15, 19, and 24. Peripheral blood and BAL samples were collected at 3, 7, 11, 15, 19, and 24 weeks after $M$. tuberculosis infection. PBMCs were isolated from blood and cryopreserved for subsequent antigen-specific flow cytometric assays. BAL samples were obtained by bronchoscopy as previously described $(38,81)$, using 2 washes of $40 \mathrm{~mL}$ sterile saline, and used for antigen-specific assays and to measure CFU. Bacterial burden associated with $M$. tuberculosis infection was determined in BAL and in lung at necropsy by plating BAL or homogenized tissue sections as previously described $(42,45,82,83)$. Individual lung lobes were cut into 2-mm thick slabs and stereologically selected for analysis, which allows for unbiased selection of lung tissue (84). Approximately $50 \%$ of the lung tissue was pooled by lung lobe ( $n=5 /$ animal), homogenized, serially diluted, and plated in quadruplicate for quantification of bacterial load by CFU. Approximately $30 \%$ of the lung tissue was fixed for histological analysis, and the remaining tissue was fixed for use in immunohistochemistry and immunofluorescence microscopy. The extent of morphometric lung pathology and the involvement of lung in granulomatous lesions was also determined at necropsy. Animals were euthanized at 24 weeks or upon signs of disease. Humane endpoints were predefined in the IACUC protocol and applied as needed to reduce discomfort (45). All animals were routinely cared for according to the guidelines prescribed by the NIH Guide for the Care and Use of Laboratory Animals (National Academies Press, 2011). 
Antigen-specific assays and flow cytometry of PBMC and BAL samples. Cell preparation tubes (CPT, BD Biosciences) were used for PBMC isolation (85), and PBMCs were cryopreserved in $90 \%$ fetal FBS (Hyclone) and $10 \%$ dimethyl sulfoxide (MilliporeSigma) until subsequent batch testing by ICS and flow cytometry. For processing of BAL, mononuclear cells were isolated by passing through a $70-\mu \mathrm{M}$ nylon cell strainer (Becton Dickinson Discovery Labware) followed by washing in complete medium (RPMI-1640 containing $10 \%$ FBS, $2 \mathrm{mM}$ glutamine, $100 \mathrm{IU} / \mathrm{mL}$ penicillin, and $100 \mu \mathrm{g} / \mathrm{mL}$ streptomycin, Lonza). Isolated BAL cells $\left(\sim 1 \times 10^{6}\right.$ to $\left.2 \times 10^{6}\right)$ were stimulated with CW antigen $(10 \mu \mathrm{g} / \mathrm{mL}$, BEI Resources $)$ and ESAT-6/CFP10 peptide pools $(10 \mu \mathrm{g} / \mathrm{mL}, 15$-mers with 11-amino acid overlap, Genemed Synthesis Inc.) for 2 hours at $37^{\circ} \mathrm{C}, 5 \% \mathrm{CO}_{2}$. Brefeldin A was added (MilliporeSigma) and cells were further incubated for 4 hours at $37^{\circ} \mathrm{C}, 5 \% \mathrm{CO}_{2}$. The stimulated BAL cells were cryopreserved using $90 \%$ fetal FBS and $10 \%$ dimethyl sulfoxide and stored in liquid nitrogen until subsequent batch processing for ICS staining and flow cytometry.

ICS and flow cytometry. Cryopreserved PBMCs were thawed (all time points for each animal were thawed on the same day) and rested overnight at $37^{\circ} \mathrm{C}, 5 \% \mathrm{CO}_{2}$, in $10 \%$ complete RPMI. PBMCs $\left(\sim 1 \times 10^{6}\right.$ to $\left.2 \times 10^{6}\right)$ were stimulated with $M$. tuberculosis $\mathrm{CW}$ antigens $(10 \mu \mathrm{g} / \mathrm{mL}$, BEI Resources $)$ or ESAT-6/CFP10 peptide pools $(10 \mu \mathrm{g} / \mathrm{mL}, 15$-mers with 11 -amino acid overlap, Genemed Synthesis Inc.) for 2 hours followed by the addition of Brefeldin $\mathrm{A}(10 \mu \mathrm{g} / \mathrm{mL})$ (BD Biosciences), after which the cells were further incubated for 16 hours. ICS and flow cytometry was performed as described below. Because of low cell yield and to maintain optimal viability, fresh cells from BAL were stimulated for 6 hours, cryopreserved, and subsequently batch processed as follows: cryopreserved, stimulated BAL cells were thawed (all time points for each animal were thawed on the same day) and processed for ICS and flow cytometry. Stimulated PBMCs and BAL cells were stained for dead cells using the LIVE/DEAD Fixable Near-IR Dead Cell Stain (Life Technologies, Thermo Fisher Scientific) and then surface stained with the following antibodies: CD8-V500 (clone SK1), CCR6- BV711 (clone 11A9), CD95-PETR (clone DX2) from BD Biosciences, CD38-FITC (clone AT-1, STEMCELL Technologies), CD28-PE-Cy7 (clone CD28.2), and CXCR3-BV605 (clone G025H7) from BioLegend. Cells were permeabilized with Cytofix/Cytoperm Kit (BD Biosciences) and stained intracellularly with CD3-PerCP (clone SP34-2), IFN- $\gamma$-Alexa Fluor 700 (clone B27), and IL-17-APC (clone MQ1-17H12) from BD Biosciences and CD4 BV650 (clone OKT4, BioLegend). Cell were fixed with $1 \%$ paraformaldehyde before acquisition in an LSR II flow cytometer (BD Biosciences). Flow cytometry data were analyzed using FlowJo software V10 (Tree Star Inc.). Unstimulated controls were used to set the gate for $\mathrm{T}$ cells expressing cytokines.

Histology and quantification of granulomatous areas in the lung. Lung tissues were fixed in zinc-buffered formalin, processed routinely, and stained with $H \& E$. The stained tissue sections were digitally scanned with a digital slide scanner (Axio Scan.Z1, Carl Zeiss) and analyzed with computer software (Tissue Classifier, HALO, Indica Labs). Annotation regions were drawn around each tissue section on the slide. Annotated regions were then classified using an algorithm trained via a deep convolutional network (HALO AI) to identify granulomas. All analyses were reviewed by a board-certified veterinary pathologist to confirm their accuracy. Percentage of granuloma within tissue sections was determined by dividing the granuloma area $\left(\mathrm{mm}^{2}\right)$ by the area of the annotated regions $\left(\mathrm{mm}^{2}\right)$.

Immunohistochemistry and confocal microscopy. In addition to tissue sections from the 6 asymptomatic animals, we included archived formalin-fixed, paraffin-embedded lung tissues from animals with active TB that were reported in our previous studies (42) and performed immunostaining and confocal microscopy as previously described (45). Briefly, 5- $\mu \mathrm{m}$ tissues sections were mounted on Superfrost Plus Microscope slides, baked overnight at $56^{\circ} \mathrm{C}$, and passed through xylene, graded ethanol, and double-distilled water to remove paraffin and rehydrate tissue sections. A microwave was used for heat-induced epitope retrieval. Slides were boiled for 20 minutes in a Tris-based solution, $\mathrm{pH} 9$ (Vector Laboratories H-3301), containing $0.01 \%$ Tween-20. Slides were briefly rinsed in hot distilled water and transferred to a hot citrate-based solution, $\mathrm{pH} 6.0$ (Vector Laboratories H-3300), where they were allowed to cool to room temperature. Once cool, slides were rinsed in Tris-buffered saline (TBS) and placed in a black humidifying chamber, where they were incubated with Background Punisher (Biocare Medical BP974H) for 10 minutes and washed with TBS containing $0.01 \%$ Triton X-100 for 5 minutes, followed by a quick rinse in TBS before being returned to the black chamber to be incubated with serum-free protein block (Dako X0909) for 20 minutes. The slides were stained with primary antibodies against the following proteins: CD68 (1:20, mouse IgG1, Dako), CD163 (1:20, mouse IgG1, Leica Biosystems), CXCR3 (1:20 mouse IgG1, BD Pharmingen), CD4 (1:20, rabbit, Abcam), and DAPI nuclear stain (1:20,000, Invitrogen, Thermo Fisher 
Scientific). The above primary antibodies were detected with the following secondary antibodies from Molecular Probes, Thermo Fisher Scientific, at a 1:1000 concentration derived from goat: goat anti-mouse IgG1 488 (green), goat anti-mouse IgG1 488 (green), Permanent Red, and goat anti-rabbit 647 (far red). Imaging was performed with a Zeiss Axio Slide Scanner (Carl Zeiss), and the images were analyzed with computer software (Hiplex FL, HALO, Indica Labs).

Quantification of $\mathrm{CXCR}^{+}$cells by immunofluorescence microscopy. Tissue segmentation was first performed using pattern recognition software (Tissue Classifier, HALO, Indica Labs). A random forest classifier was set at a resolution of $7 \mu \mathrm{m} /$ pixel and to detect a minimum object size of $50 \mu \mathrm{m}^{2}$. The classifier was then trained to detect the following tissue classes by being provided multiple examples of each tissue class: granuloma and nongranuloma. Annotation regions were drawn around each piece of tissue on a slide, and tissue segmentation was performed on the entire piece of tissue (Supplemental Figure 5). Computer software (Hiplex FL, HALO, Indica Labs) was used to quantify the following phenotypes: CD4 ${ }^{+}$ cells (blue), $\mathrm{CXCR}^{+}$cells (red), $\mathrm{CD}^{+} 8^{+} / \mathrm{CD} 163^{+}$macrophages (green), and CD4 ${ }^{+} \mathrm{CXCR} 3^{+}$cells (purple). The software used thresholding to both detect cells and set cutoff values for expression of each marker/ channel. Thresholds were set by real-time visual assessment of known positive and negative cells. Analysis and quantification of cellular phenotypes were performed on each tissue segment as defined above.

Statistics. Statistical analyses were performed using GraphPad Prism (GraphPad Software). The specific tests used are indicated in each of the figure legends. $P<0.05$ was considered statistically significant.

Study approval. All animal procedures were approved by the IACUC and by the Institutional Biosafety Committee of Tulane University, New Orleans, Louisiana, USA, and were performed in strict accordance with NIH guidelines. The TNPRC facilities are accredited by the American Association for Accreditation of Laboratory Animal Care and licensed by the U.S. Department of Agriculture.

\section{Author contributions}

JR, DK, and US conceived the studies. US, ANB, SRG, MQ, CI, XA, and RVB performed experiments. US, JR, DK, ANB, CI, XA, and VV performed data analyses. US, JR, DK, and VV wrote the manuscript.

\section{Acknowledgments}

We thank Toidi Adekambi for help with PBMC processing and optimization of flow cytometry assays and Lakshmi Chennareddi for help with statistical analysis. We also thank present lab members for their helpful suggestions and Jonathan Kevin Sia for comments on the manuscript. We acknowledge the funding support from the following NIH grants: AI050409, R01AI111943, R01AI123047, R01AI134240, P51OD011133, P51OD011132, and P51OD011104.

Address correspondence to: Jyothi Rengarajan, Emory Vaccine Center at the Yerkes National Primate Center, Emory University School of Medicine, 954 Gatewood Road, Atlanta, Georgia 30329, USA. Phone: 404.727.8174; jrengar@emory.edu. Or to: Deepak Kaushal, Southwest National Primate Research Center, Texas Biomedical Research Institute, 8715 W. Military Drive, San Antonio, Texas 78227, USA. Phone; 210.258.9445; Email: kaushal@txbiomed.org. ANB's present address is: Vaccine Research Center, National Institute of Allergy and Infectious Diseases, NIH, Bethesda, Maryland, Bethesda, Maryland, USA.

1. WHO. Latent tuberculosis infection: updated and consolidated guidelines for programmatic management. WHO. https://www.who.int/ tb/publications/2018/latent-tuberculosis-infection/en/. 2018. Accessed June 24, 2020

2. Tait DR, et al. Final analysis of a trial of M72/AS01 vaccine to prevent tuberculosis. NEngl J Med. 2019;381(25):2429-2439.

3. Barry CE, et al. The spectrum of latent tuberculosis: rethinking the biology and intervention strategies. Nat Rev Microbiol. 2009;7(12):845-855.

4. Lin PL, et al. PET CT identifies reactivation risk in cynomolgus macaques with latent $M$. tuberculosis. PLoS Pathog. 2016;12(7):e1005739.

5. Lin PL, Flynn JL. The end of the binary era: revisiting the spectrum of tuberculosis. J Immunol. 2018;201(9):2541-2548

6. Flynn JL, Chan J, Triebold KJ, Dalton DK, Stewart TA, Bloom BR. An essential role for interferon gamma in resistance to Mycobacterium tuberculosis infection. J Exp Med. 1993;178(6):2249-2254.

7. Green AM, Difazio R, Flynn JL. IFN- $\gamma$ from CD4 T cells is essential for host survival and enhances CD8 T cell function during Mycobacterium tuberculosis infection. J Immunol. 2013;190(1):270-277.

8. Lyadova I, Nikitina I. Cell differentiation degree as a factor determining the role for different T-helper populations in tuberculosis protection. Front Immunol. 2019;10:972.

9. Goletti D, Petruccioli E, Joosten SA, Ottenhoff TH. Tuberculosis biomarkers: from diagnosis to protection. Infect Dis Rep. 2016;8(2):6568. 
10. Khader SA, et al. IL-23 and IL-17 in the establishment of protective pulmonary CD4 ${ }^{+} \mathrm{T}$ cell responses after vaccination and during Mycobacterium tuberculosis challenge. Nat Immunol. 2007;8(4):369-377.

11. Okamoto Yoshida Y, et al. Essential role of IL-17A in the formation of a mycobacterial infection-induced granuloma in the lung. J Immunol. 2010;184(8):4414-4422.

12. Wang M, et al. Genetic polymorphisms of IL-17A, IL-17F, TLR4 and miR-146a in association with the risk of pulmonary tuberculosis. Sci Rep. 2016;6:28586.

13. Gopal R, et al. Unexpected role for IL-17 in protective immunity against hypervirulent Mycobacterium tuberculosis HN878 infec tion. PLoS Pathog. 2014;10(5):e1004099.

14. Gideon HP, et al. Variability in tuberculosis granuloma $\mathrm{T}$ cell responses exists, but a balance of pro- and anti-inflammatory cytokines is associated with sterilization. PLoS Pathog. 2015;11(1):e1004603.

15. Freches D, Korf H, Denis O, Havaux X, Huygen K, Romano M. Mice genetically inactivated in interleukin-17A receptor are defective in long-term control of Mycobacterium tuberculosis infection. Immunology. 2013;140(2):220-231.

16. Dijkman K, et al. Prevention of tuberculosis infection and disease by local BCG in repeatedly exposed rhesus macaques. Nat Med. 2019;25(2):255-262.

17. Aguilo N, et al. Pulmonary but not subcutaneous delivery of BCG vaccine confers protection to tuberculosis-susceptible mice by an interleukin 17-dependent mechanism. J Infect Dis. 2016;213(5):831-839.

18. Sia JK, Bizzell E, Madan-Lala R, Rengarajan J. Engaging the CD40-CD40L pathway augments T-helper cell responses and improves control of Mycobacterium tuberculosis infection. PLoS Pathog. 2017;13(8):e1006530.

19. Dheda K, Chang JS, Lala S, Huggett JF, Zumla A, Rook GA. Gene expression of IL17 and IL23 in the lungs of patients with active tuberculosis. Thorax. 2008;63(6):566-568.

20. Scriba TJ, et al. Distinct, specific IL-17- and IL-22-producing CD4 ${ }^{+} \mathrm{T}$ cell subsets contribute to the human anti-mycobacterial immune response. J Immunol. 2008;180(3):1962-1970.

21. Chen YC, et al. Prognostic values of serum IP-10 and IL-17 in patients with pulmonary tuberculosis. Dis Markers. 2011;31(2):101-110

22. Okada S, et al. Immunodeficiencies. Impairment of immunity to Candida and Mycobacterium in humans with bi-allelic RORC mutations. Science. 2015;349(6248):606-613.

23. Singh S, et al. Interleukin-17 regulates matrix metalloproteinase activity in human pulmonary tuberculosis. $J$ Pathol. 2018;244(3):311-322.

24. Cooper AM. IL-17 and anti-bacterial immunity: protection versus tissue damage. Eur J Immunol. 2009;39(3):649-652.

25. Basaraba RJ. Experimental tuberculosis: the role of comparative pathology in the discovery of improved tuberculosis treatment strategies. Tuberculosis (Edinb). 2008;88(supp1 1):S35-S47.

26. Basile JI, et al. Outbreaks of Mycobacterium tuberculosis MDR strains induce high IL-17 T-cell response in patients with MDR tuberculosis that is closely associated with high antigen load. J Infect Dis. 2011;204(7):1054-1064

27. Zhao HM, et al. Differential T cell responses against DosR-associated antigen Rv2028c in BCG-vaccinated populations with tuberculosis infection. J Infect. 2019;78(4):275-280.

28. Lu LL, et al. IFN- $\gamma$-independent immune markers of Mycobacterium tuberculosis exposure. Nat Med. 2019;25(6):977-987.

29. Essone PN, et al. M. tuberculosis infection and antigen specific cytokine response in healthcare workers frequently exposed to tuberculosis. Sci Rep. 2019;9(1):8201.

30. Schuck SD, et al. Identification of T-cell antigens specific for latent Mycobacterium tuberculosis infection. PLoS One. 2009;4(5):e5590.

31. Barham MS, et al. HIV Infection is associated with downregulation of BTLA expression on Mycobacterium tuberculosis-specific CD4 T cells in active tuberculosis disease. Front Immunol. 2019;10:1983.

32. Chiacchio T, et al. Higher frequency of T-cell response to $M$. tuberculosis latency antigen Rv2628 at the site of active tuberculosis disease than in peripheral blood. PLoS One. 2011;6(11):e27539.

33. Jarvela JR, Tuscano L, Lee H, Silver RF. Pulmonary responses to pathogen-specific antigens in latent Mycobacterium tuberculosis infection. Tuberculosis (Edinb). 2016;96:158-164.

34. Jarvela J, et al. Mycobacterium tuberculosis-induced bronchoalveolar lavage gene expression signature in latent tuberculosis infection is dominated by pleiotropic effects of $\mathrm{CD}^{+} \mathrm{T}$ cell-dependent IFN- $\gamma$ production despite the presence of polyfunctional $\mathrm{T}$ cells within the airways. J Immunol. 2019;203(8):2194-2209.

35. Capuano SV, et al. Experimental Mycobacterium tuberculosis infection of cynomolgus macaques closely resembles the various manifestations of human M. tuberculosis infection. Infect Immun. 2003;71(10):5831-5844.

36. Walsh GP, et al. The Philippine cynomolgus monkey (Macaca fasicularis) provides a new nonhuman primate model of tuberculosis that resembles human disease. Nat Med. 1996;2(4):430-436.

37. Lin PL, et al. Quantitative comparison of active and latent tuberculosis in the cynomolgus macaque model. Infect Immun. 2009;77(10):4631-4642.

38. Mehra S, et al. Reactivation of latent tuberculosis in rhesus macaques by coinfection with simian immunodeficiency virus J Med Primatol. 2011;40(4):233-243.

39. Foreman TW, et al. Isoniazid and rifapentine treatment eradicates persistent Mycobacterium tuberculosis in macaques. Am J Respir Crit Care Med. 2020;201(4):469-477.

40. Bucşan AN, et al. Mechanisms of reactivation of latent tuberculosis infection due to SIV coinfection. J Clin Invest. 2019;129(12):5254-5260.

41. Bucsan AN, Rout N, Foreman TW, Khader SA, Rengarajan J, Kaushal D. Mucosal-activated invariant T cells do not exhibit significant lung recruitment and proliferation profiles in macaques in response to infection with Mycobacterium tuberculosis CDC1551. Tuberculosis (Edinb). 2019;116S:S11-S18

42. Foreman TW, et al. CD4 ${ }^{+}$T-cell-independent mechanisms suppress reactivation of latent tuberculosis in a macaque model of HIV coinfection. Proc Natl Acad Sci U S A. 2016;113(38):E5636-E5644.

43. Sallusto F, Lenig D, Förster R, Lipp M, Lanzavecchia A. Two subsets of memory T lymphocytes with distinct homing potentials and effector functions. Nature. 1999;401(6754):708-712. 
44. Lindenstrøm T, et al. Tuberculosis subunit vaccination provides long-term protective immunity characterized by multifunctional CD4 memory T cells. J Immunol. 2009;182(12):8047-8055.

45. Kaushal D, et al. Mucosal vaccination with attenuated Mycobacterium tuberculosis induces strong central memory responses and protects against tuberculosis. Nat Commun. 2015;6:8533.

46. Pitcher CJ, et al. Development and homeostasis of T cell memory in rhesus macaque. J Immunol. 2002;168(1):29-43.

47. Groom JR, Luster AD. CXCR3 in T cell function. Exp Cell Res. 2011;317(5):620-631.

48. Kim CH. Migration and function of Th17 cells. Inflamm Allergy Drug Targets. 2009;8(3):221-228.

49. Ito T, Carson WF, Cavassani KA, Connett JM, Kunkel SL. CCR6 as a mediator of immunity in the lung and gut. Exp Cell Res. 2011;317(5):613-619.

50. Kuroda MJ, et al. High turnover of tissue macrophages contributes to tuberculosis reactivation in simian immunodeficiency virus-infected rhesus macaques. J Infect Dis. 2018;217(12):1865-1874.

51. Mehra S, et al. Granuloma correlates of protection against tuberculosis and mechanisms of immune modulation by Mycobacterium tuberculosis. J Infect Dis. 2013;207(7):1115-1127.

52. Lin PL, et al. Early events in Mycobacterium tuberculosis infection in cynomolgus macaques. Infect Immun. 2006;74(7):3790-3803.

53. Foreman TW, Mehra S, Lackner AA, Kaushal D. Translational research in the nonhuman primate model of tuberculosis. ILAR J. 2017;58(2):151-159.

54. Bucsan AN, Mehra S, Khader SA, Kaushal D. The current state of animal models and genomic approaches towards identifying and validating molecular determinants of Mycobacterium tuberculosis infection and tuberculosis disease. Pathog Dis. 2019;77(4):ftz037.

55. Shen $\mathrm{L}$, et al. Immunization of $\mathrm{V} \gamma 2 \mathrm{~V} \delta 2 \mathrm{~T}$ cells programs sustained effector memory responses that control tuberculosis in nonhuman primates. Proc Natl Acad Sci U S A. 2019;116(13):6371-6378.

56. Flynn JL, et al. Non-human primates: a model for tuberculosis research. Tuberculosis (Edinb). 2003;83(1-3):116-118.

57. White AG, et al. Analysis of 18FDG PET/CT imaging as a tool for studying Mycobacterium tuberculosis infection and treatment in non-human primates. J Vis Exp. 2017;(127):56375.

58. Maiello P, et al. Rhesus macaques are more susceptible to progressive tuberculosis than cynomolgus macaques: a quantitative comparison. Infect Immun. 2018;86(2):e0505-17.

59. Mattila JT, Diedrich CR, Lin PL, Phuah J, Flynn JL. Simian immunodeficiency virus-induced changes in T cell cytokine responses in cynomolgus macaques with latent Mycobacterium tuberculosis infection are associated with timing of reactivation. J Immunol. 2011;186(6):3527-3537.

60. Lin PL, et al. Sterilization of granulomas is common in active and latent tuberculosis despite within-host variability in bacterial killing. Nat Med. 2014;20(1):75-79.

61. Kauffman KD, et al. Defective positioning in granulomas but not lung-homing limits CD4 T-cell interactions with Mycobacterium tuberculosis-infected macrophages in rhesus macaques. Mucosal Immunol. 2018;11(2):462-473.

62. Diedrich CR, et al. CD4CD8 Double positive T cell responses during Mycobacterium tuberculosis infection in cynomolgus macaques. J Med Primatol. 2019;48(2):82-89.

63. Silver RF, et al. Diversity of human and macaque airway immune cells at baseline and during tuberculosis infection. Am J Respir Cell Mol Biol. 2016;55(6):899-908.

64. Darrah PA, et al. Aerosol vaccination with AERAS-402 elicits robust cellular immune responses in the lungs of rhesus macaques but fails to protect against high-dose Mycobacterium tuberculosis challenge. J Immunol. 2014;193(4):1799-1811.

65. Darrah PA, et al. Boosting BCG with proteins or rAd5 does not enhance protection against tuberculosis in rhesus macaques. NPJ Vaccines. 2019;4:21.

66. Darrah PA, et al. Prevention of tuberculosis in macaques after intravenous BCG immunization. Nature. 2020;577(7788):95-102

67. Iwanaga N, Kolls JK. Updates on T helper type 17 immunity in respiratory disease. Immunology. 2019;156(1):3-8

68. Van Dis E, et al. STING-Activating adjuvants elicit a Th17 immune response and protect against Mycobacterium tuberculosis infection. Cell Rep. 2018;23(5):1435-1447.

69. Gallegos AM, van Heijst JW, Samstein M, Su X, Pamer EG, Glickman MS. A gamma interferon independent mechanism of CD4 T cell mediated control of M. tuberculosis infection in vivo. PLoS Pathog. 2011;7(5):e1002052.

70. Wareham AS, Tree JA, Marsh PD, Butcher PD, Dennis M, Sharpe SA. Evidence for a role for interleukin-17, Th17 cells and iron homeostasis in protective immunity against tuberculosis in cynomolgus macaques. PLoS One. 2014;9(2):e88149.

71. Orme IM. Pillars article: the kinetics of emergence and loss of mediator T lymphocytes acquired in response to infection with Mycobacterium Tuberculosis. J. Immunol. 1987. 138: 293-298. J Immunol. 2017;199(3):833-838.

72. Bluestone JA, Mackay CR, O'Shea JJ, Stockinger B. The functional plasticity of T cell subsets. Nat Rev Immunol. 2009;9(11):811-816.

73. Acosta-Rodriguez EV, et al. Surface phenotype and antigenic specificity of human interleukin 17-producing T helper memory cells. Nat Immunol. 2007;8(6):639-646.

74. Ramesh R, et al. Pro-inflammatory human Th17 cells selectively express P-glycoprotein and are refractory to glucocorticoids. J Exp Med. 2014;211(1):89-104.

75. Silveira-Mattos PS, et al. Differential expression of CXCR3 and CCR6 on CD4 ${ }^{+}$T-lymphocytes with distinct memory phenotypes characterizes tuberculosis-associated immune reconstitution inflammatory syndrome. Sci Rep. 2019;9(1):1502

76. Lindestam Arlehamn CS, et al. Memory T cells in latent Mycobacterium tuberculosis infection are directed against three antigenic islands and largely contained in a CXCR3 ${ }^{+}$CCR6 ${ }^{+}$Th1 subset. PLoS Pathog. 2013;9(1):e1003130.

77. Arlehamn CL, et al. Transcriptional profile of tuberculosis antigen-specific T cells reveals novel multifunctional features J Immunol. 2014;193(6):2931-2940.

78. Goldberg MF, et al. Salmonella persist in activated macrophages in T cell-sparse granulomas but are contained by surrounding CXCR3 ligand-positioned Th1 cells. Immunity. 2018;49(6):1090-1102.e7.

79. Hartings JM, Roy CJ. The automated bioaerosol exposure system: preclinical platform development and a respiratory dosimetry application with nonhuman primates. J Pharmacol Toxicol Methods. 2004;49(1):39-55.

80. Corleis B, et al. HIV-1 and SIV infection are associated with early loss of lung interstitial CD $4^{+} \mathrm{T}$ cells and dissemination of 
pulmonary tuberculosis. Cell Rep. 2019;26(6):1409-1418.e5.

81. Mehra S, et al. The DosR regulon modulates adaptive immunity and is essential for Mycobacterium tuberculosis persistence. Am J Respir Crit Care Med. 2015;191(10):1185-1196.

82. Kaushal D, Mehra S, Didier PJ, Lackner AA. The non-human primate model of tuberculosis. J Med Primatol. 2012;41(3):191-201.

83. Dutta NK, McLachlan J, Mehra S, Kaushal D. Humoral and lung immune responses to Mycobacterium tuberculosis infection in a primate model of protection. Trials Vaccinol. 2014;3:47-51.

84. Luciw PA, et al. Stereological analysis of bacterial load and lung lesions in nonhuman primates (rhesus macaques) experimentally infected with Mycobacterium tuberculosis. Am J Physiol Lung Cell Mol Physiol. 2011;301(5):L731-L738.

85. Adekambi T, et al. Biomarkers on patient $\mathrm{T}$ cells diagnose active tuberculosis and monitor treatment response. J Clin Invest. 2015;125(5):1827-1838 